\title{
MOTIVIC SERRE INVARIANTS, RAMIFICATION, AND THE ANALYTIC MILNOR FIBER
}

\author{
JOHANNES NICAISE* AND JULIEN SEBAG
}

\section{INTRODUCTION}

Let us recall the classical definition of a $p$-adic zeta function, as it was given by Igusa [29]. A survey of the theory of $p$-adic zeta functions can be found in Denef's Bourbaki report [19].

In its simplest form, a $p$-adic zeta function is defined by the $p$-adic integral

$$
Z_{p}(f, s)=\int_{\mathbb{Z}_{p}^{n}}|f(x)|^{s}|d x|,
$$

where $s$ is a complex variable, $f$ is a polynomial over $\mathbb{Z}_{p}$ in $n$ variables, $|f|$ is its $p$-adic norm, and $|d x|$ denotes the Haar measure on the compact group $\mathbb{Z}_{p}^{n}$, normalized to give $\mathbb{Z}_{p}^{n}$ measure 1 . A priori, $Z_{p}(f, s)$ is only defined when $\Re(s)>0$. However, Igusa proved, using resolution of singularities, that it has a meromorphic continuation to the complex plane. Moreover, it is a rational function in $p^{-s}$. The numerical data of an embedded resolution for $f$ yield a complete set of candidate poles of $Z_{p}(f, s)$, but, since this set depends on the chosen resolution, a lot of these candidate poles will not be actual poles of $Z_{p}(f, s)$. The $p$-adic zeta function $Z_{p}(f, s)$ can be written as a Mellin transform of the local singular series of $f$ (defined by integrating the Gelfand-Leray form $d x / d f$ on the regular fibers of $f$ ); see [19, 1.4].

When $f$ is defined over $\mathbb{Q}$, Igusa's Monodromy Conjecture predicts an intriguing connection between the eigenvalues of the monodromy at complex points of $f^{-1}(0) \subset \mathbb{C}^{n}$, and the poles of $Z_{p}(f, s)$, for almost all primes $p$, where $f$ is considered as a complex, respectively $p$-adic polynomial. Since the $p$-adic zeta function contains information about the number of solutions of the congruence $f(x) \equiv 0$ modulo powers of $p$, the monodromy conjecture establishes a fascinating bridge between arithmetic properties and complex topology, much like the Weil conjectures. In fact, we will show in this paper that this analogy is more than just philosophical.

In order to geometrize the situation, Denef and Loeser introduced the topological zeta function $Z_{t o p}(f, s)$, which is some kind of geometric abstraction of the $p$-adic zeta function. It is a rational function in the complex variable $s$, defined in terms of an embedded resolution of singularities, and to show that the definition does not depend on the choice of resolution, Denef and Loeser established $Z_{t o p}$ as a formal limit of $p$-adic zeta functions. After Kontsevich introduced motivic integration 32 , it became clear that both the $p$-adic and the topological zeta function are avatars of a universal being, the "naïve" motivic zeta function $Z_{m o t}(f, s)$ (the more exact statement says that $Z_{m o t}$ specializes to $Z_{t o p}$, and to $Z_{p}$ for almost all primes

* During part of the research for this article, the first author was Research Assistant of the Fund for Scientific Research - Flanders (Belgium)(F.W.O.) at the Department of Mathematics of the Katholieke Universiteit Leuven (Belgium). 
$p$ ). This naïve motivic zeta function is defined intrinsically by means of a motivic integral, but it can be expressed explicitly in terms of an embedded resolution of singularities for the morphism $f$. In [23, 3.5.3], Denef and Loeser define the motivic nearby cycles of $f$ as a limit of the motivic zeta function. It is an object in an appropriate Grothendieck ring of varieties over the complex hypersurface $X_{s}=f^{-1}(0)$, and the fiber over each point $x \in X_{s}$ has the same Hodge polynomial as the Milnor fiber of $f$ at $x$. The motivic monodromy conjecture states that each pole of the naïve motivic zeta function induces an eigenvalue of the monodromy at some point of $X_{s}$. In this setting, the notion of pole has to be defined with care [41, §4], since the Grothendieck ring of varieties is not a domain [40.

Most research on this conjecture made a "detour" via a resolution of singularities, studying the geometry of the exceptional locus in order to eliminate fake candidate poles, and applying A'Campo's formula for the monodromy zeta function [4]. This approach has yielded proofs in particular cases, and inspired nice results concerning the geometry of embedded resolutions, but it seems difficult to apply this technique to the general case. In this article, we try to establish a more direct link between the motivic zeta function of $f$, and the Milnor fibration, using Berkovich' étale cohomology for analytic spaces [6], and the theory of motivic integration on rigid spaces 34. More precisely, we introduce the analytic Milnor fiber, a rigid variety over $\mathbb{C}((t))$ with the "same" cohomology as the topological Milnor fiber, and whose points are closely related to the arc spaces used to define the motivic zeta function.

We briefly sketch the ideas of the construction. Let $f$ be a non-constant complex polynomial in $n$ variables, and let $x$ be a closed point of the hypersurface $X_{s}$ defined by $f$. Let $B=B(x, \varepsilon)$ be a small open disc around $x$ in $\mathbb{C}^{n}$, and let $D=B(0, \eta)$ be a small open disc around the origin in $\mathbb{C}$. We denote $D-0$ by $D^{*}$, and we denote by $\widetilde{D^{*}} \rightarrow D^{*}$ the universal covering space

$$
\widetilde{D^{*}}=\{z \in \mathbb{C} \mid \operatorname{Im}(z)>-\log (\eta)\} \rightarrow D^{*}: z \mapsto \exp (i z)
$$

For $0<\eta \ll \varepsilon \ll 1$, the mapping

$$
f: B \cap f^{-1}\left(D^{*}\right) \rightarrow D^{*}
$$

is a locally trivial fibration, called the Milnor fibration of $f$ at $x$. Its canonical fiber $\left(B \cap f^{-1}\left(D^{*}\right)\right) \times_{D^{*}} \widetilde{D^{*}}$ is called the canonical topological Milnor fiber $F_{x}$ of $f$ at $x$. The group of deck transformations $\pi_{1}\left(D^{*}\right)$ acts on $F_{x}$, and the canonical generator $z \mapsto z+2 \pi$ of $\pi_{1}\left(D^{*}\right)$ induces a monodromy automorphism on the singular cohomology spaces $H_{\text {sing }}^{i}\left(F_{x}, \mathbb{C}\right)$. The Milnor fibration is used as a tool to gather information about the topology of $X_{s}$ near $x$ (see [35]).

We can mimic this construction in the setting of formal geometry. Let $k$ be an algebraically closed field of characteristic zero, and let $f$ be a dominant morphism $f: X \rightarrow$ Spec $k[t]$, with $X$ a smooth irreducible variety over $k$. Denote by $X_{s}$ the hypersurface defined by $f$. We put $R=k[[t]]$, and $K=k((t))$. Moreover, for each integer $d>0$, we denote by $K(d)$ the unique extension $k((\sqrt[d]{t}))$ of degree $d$ over $K$, and we denote by $R(d)$ the normalization $k[[\sqrt[d]{t}]]$ of $R$ in $K(d)$.

Taking the formal $t$-adic completion of $f$, we obtain a formal $R$-scheme $\widehat{X}$, whose special fiber is isomorphic to $X_{s}$. The "complement" of $X_{s}$ in $\widehat{X}$ is a smooth rigid variety $X_{\eta}$ over $K$, and is endowed with a canonical specialization map of ringed sites $s p: X_{\eta} \rightarrow \widehat{X}$; see [8, 0.2]. 
As we'll see in Section 9.1.2, for any integer $d>0, K(d)$-valued points on $X_{\eta}$ correspond canonically to $\operatorname{arcs} \psi: \operatorname{Spec} R \rightarrow X$ on $X$, with $f(\psi)=t^{d}$. The specialization morphism $s p$ maps $\psi$ to its origin $\psi(0)$. In Section 9.1, we define the analytic Milnor fiber of $f$ at a closed point $x$ of $X_{s}$ as $s p^{-1}(x)$, and we denote it by $\mathscr{F}_{x}$. It is an open rigid subspace of $X_{\eta}$. If $k=\mathbb{C}$, we prove in Section 9.1.1 that its $\ell$-adic cohomology is isomorphic to the singular cohomology of the topological Milnor fiber $F_{x}$, and the absolute Galois action of $G\left(K^{s} / K\right)$ corresponds to the action of the monodromy.

We define the local singular series $F(f ; d)$ of $f$ by integrating the Gelfand-Leray form associated to $f$ on $X_{\eta} \times_{K} K(d)$ (Definition 9.5 and 9.8, the definition of the motivic integral of a volume form on a smooth rigid variety is recalled in Subsection 6.1). The result is a function $F(f ;$.$) from \mathbb{N}^{*}$ to the localized Grothendieck ring $\mathcal{M}_{X_{s}}$. Theorem 9.9 states that the motivic zeta function coincides (modulo a factor $\mathbb{L})$ with the Mellin transform $\sum_{d>0} F(f ; d) T^{d}$.

Hence, we recover the motivic zeta function as a Weil-type zeta function of the "nearby fiber" $X_{\eta}$. The corresponding cohomological interpretation in Theorem 9.11 generalizes a result by Denef and Loeser [24, 1.1] which expresses the Lefschetz numbers of the monodromy transformation, in terms of the Euler characteristic of the jet spaces of the hypersurface defined by $f$. We will obtain it as a special case of our Theorem 5.4 a Lefschetz trace formula for rigid spaces, which relates the Euler characteristic of the motivic Serre invariant to the Galois action on the étale cohomology.

Finally, we will prove in Section 7 that, for any generically smooth stft formal $R$-scheme $X_{\infty}$, and any gauge form $\omega$ on $X_{\eta}$, the volume Poincaré series

$$
S\left(X_{\infty}, \omega ; T\right):=\left(\sum_{d>0} \int_{X_{\eta} \times_{K} K(d)}\left|\omega \otimes_{K} K(d)\right|\right) T^{d} \in \mathcal{M}_{X_{s}}[[T]]
$$

is rational over $\mathcal{M}_{X_{s}}$, and we give an explicit expression. This allows us to associate a motivic volume to $X_{\eta} \widehat{\times}_{K} \widehat{K^{s}}$ in Section 8 . When $X_{\infty}$ is the completion of a morphism $f: X \rightarrow \mathbb{A}_{k}^{1}$ as above, this motivic volume coincides with Denef and Loeser's motivic nearby cycles, as we will see in Section 9.4

To conclude this introduction, we give a survey of the structure of this paper. In Section 2, we recall some basic facts on formal schemes and rigid varieties. Moreover, we prove resolution of singularities for affine, flat, generically smooth formal $k[[t]]$-schemes if $k$ has characteristic zero (Proposition 2.5). In Section 3 , we recall the definition of the (relative) motivic Serre invariant, as introduced in 39, and we briefly discuss motivic Serre invariants with support. In Section 4, we construct weak Néron models for the tame ramifications of a regular flat st $f t$ formal $R$-scheme with strict normal crossings, where $R$ is a complete discrete valuation ring with perfect residue field (Theorem 4.5). Our trace formula for rigid spaces is proved in Section 5 (Theorem [5.4). In Section 6, we briefly recall the definition of the motivic integral of a gauge form on a smooth rigid variety. Next, we define the order of a gauge form $\omega$ on the generic fiber of a regular stft formal $k[[t]]$-scheme $X_{\infty}$ with strict normal crossings, assuming that $k$ has characteristic zero (Definition 6.8), and we prove its main properties. We compute the volume Poincaré series of the pair $\left(X_{\infty}, \omega\right)$ in Section 7 (Theorem 7.6). The coefficients of this Poincaré series are the motivic integrals of $\omega$ over the ramifications $X_{\eta} \times_{K} K(d)$ (Definition 7.2). The explicit expression shows that the series is rational over the localized 
Grothendieck ring $\mathcal{M}_{X_{s}}$, and allows us to define the motivic volume of $X_{\infty}$ in Section 8 as a limit of this Poincaré series (Definition 8.3).

Finally, Section 9 contains the applications to the theory of motivic zeta functions. In Subsection 9.1 we define the analytic Milnor fiber of a morphism of algebraic varieties $f: X \rightarrow \mathbb{A}_{k}^{1}$, and we study its étale cohomology and its points, in comparison to the the singular cohomology of the topological Milnor fiber (if $k=\mathbb{C}$ ), and the points of the arc space of $X$. In Subsection 9.2, we define the local singular series of $f$, and we give an explicit expression in terms of an embedded resolution of singularities for $f$. In Subsection 9.3 we show that the Mellin transform of the local singular series coincides with the motivic zeta function of $f$ (Theorem 9.10). To conclude, in Subsection 9.4, we compare the motivic volume of the rigid nearby fiber to Denef and Loeser's motivic nearby cycles.

The results in this paper were announced in [37. A survey of the philosophy behind the project can be found in [36].

\section{Preliminaries}

2.1. Notation and conventions. Throughout this paper, $R$ will be a complete discrete valuation ring, with quotient field $K$, and perfect residue field $k$. We denote the characteristic exponent of $k$ by $p$. We fix a uniformizing element $t$ in $R$, i.e. a generator of the maximal ideal.

For any integer $d>0$, prime to $p$, we denote by $K(d)$ the totally ramified extension $K\left[t_{d}\right] /\left(\left(t_{d}\right)^{d}-t\right)$ of $K$, and by $R(d)$ the normalization of $R$ in $K(d)$. For any $R$-scheme $X$, for any formal $R$-scheme $X_{\infty}$, and for any integer $d>0$ prime to $p$, we write $X(d):=X \times_{R} R(d)$ and $X(d)_{\infty}:=X_{\infty} \times_{R} R(d)$.

For any field $F$, we will denote by $F^{s}$ a separable closure. We denote the normalization of $R$ in $K^{s}$ by $R^{s}$. The residue field $k^{s}$ of $R^{s}$ is a separable closure of $k$. We'll write $\widehat{K^{s}}$ for the completion of the valued field $K^{s}$, and $\widehat{R^{s}}$ for the closure of $R^{s}$ in $\widehat{K^{s}}$.

Furthermore, we'll denote by $K^{t}$ the maximal tamely ramified extension of $K$ in $K^{s}$, and by $R^{t}$ the normalization of $R$ in $K^{t}$. The residue field of $R^{t}$ is again equal to $k^{s}$. We'll write $\widehat{K^{t}}$ for the completion of the valued field $K^{t}$, and $\widehat{R^{t}}$ for the closure of $R^{t}$ in $\widehat{K^{t}}$.

When $\mathscr{Y}$ is a rigid space over an algebraic, tamely ramified extension $K^{\prime}$ of $K$, we denote by $\overline{\mathscr{Y}}$ the base change $\mathscr{Y} \widehat{\times}_{K^{\prime}} \widehat{K^{t}}$.

A variety over a scheme $S$ is a reduced separated $S$-scheme of finite type. We do not require it to be irreducible. For any scheme $S$, we denote the underlying reduced scheme by $S_{\text {red }}$. For any $k$-scheme $S$ of finite type, we denote by $\operatorname{Sm}(S)$ its $k$-smooth part.

For any $R$-scheme $X$, we denote its special fiber $X \times_{R} k$ by $X_{s}$. We call $X$ generically smooth, if its generic fiber $X \times_{R} K$ is a smooth $K$-scheme.

If $T$ is any topological space, we write $H_{\text {sing }}(T, \mathbb{C})$ for the graded vector space $\oplus_{i} H_{\text {sing }}^{i}(T, \mathbb{C})$, where $H_{\text {sing }}^{i}($.$) denotes the i$-th singular cohomology space.

Recall the following convention: let $V=\oplus_{i \in \mathbb{Z}} V_{i}$ be a graded vector space, with $V_{i} \neq 0$ for only a finite number of $i$, and $V_{i}$ of finite dimension for all $i$. If $F=\oplus_{i} F_{i}$ is a graded endomorphism of $V$, then the trace of $F$ on $V$ is defined as

$$
\operatorname{Tr}(F \mid V):=\sum_{i \in \mathbb{Z}}(-1)^{i} \operatorname{Tr}\left(F_{i} \mid V_{i}\right)
$$


Likewise, the dimension of $V$ is the Euler characteristic

$$
\operatorname{dim} V:=\sum_{i \in \mathbb{Z}}(-1)^{i} \operatorname{dim} V_{i}
$$

We denote by $\mathbb{N}^{*}$ the set of integers $n>0$.

2.2. Formal schemes and rigid varieties. An stft formal $R$-scheme $X_{\infty}$ is a separated formal scheme, topologically of finite type over $R$. We denote its special fiber by $X_{s}$, and its generic fiber (in the category of separated quasi-compact rigid $K$-varieties) by $X_{\eta}$. We say $X_{\infty}$ is generically smooth, if $X_{\eta}$ is a smooth rigid $K$-variety. We denote by $\operatorname{Sm}\left(X_{\infty}\right)$ the smooth part of $X_{\infty}$ over $R$. If $R^{\prime}$ is a finite extension of $R$, and $\psi$ is an element of $X_{\infty}\left(R^{\prime}\right)$, then we denote by $\psi(0)$ the image of the unique point of $\operatorname{Spf} R^{\prime}$ in $X_{s}$.

For any flat $R$-variety $X$, we denote its formal $t$-adic completion by $\widehat{X}$. It is a stft flat formal $R$-scheme, and $\widehat{X}$ is generically smooth if $X$ is. If $R=k[[t]]$, we will also write $\widehat{X}$ for the $t$-adic completion of a morphism of $k$-varieties $X \rightarrow \operatorname{Spec} k[t]$, flat over the origin.

As explained in $[8,0.2]$, there exists a canonical specialization morphism of ringed sites

$$
s p: X_{\eta} \rightarrow X_{\infty}
$$

For any locally closed subscheme $Z$ of $X_{s}$, we denote by $\widehat{X_{\infty} / Z}$ the formal completion of $X_{\infty}$ along $Z$. It is topologically of finite type over $R$ if $Z$ is open in $X_{s}$, but not in general. Moreover, $s p^{-1}(Z)$ is a rigid subspace of $X_{\eta}$, and this space is canonically isomorphic to the generic fiber of $\widehat{X_{\infty} / Z}$. We denote this rigid space by ] $Z$ [, and call it the tube of $Z$ in $X_{\infty}$. It is quasi-compact if $Z$ is open in $X_{s}$, but not in general.

2.3. Dilatations. Let $X_{\infty}$ be a stft flat formal $R$-scheme, and let $V$ be a closed subscheme of $X_{s}$, defined by an ideal sheaf $\mathcal{I}$ on $X_{\infty}$. We denote the formal blow-up of $X_{\infty}$ at $V$ by $B l_{V}: X_{\infty}^{\prime} \rightarrow X_{\infty}$. The dilatation $h: Y_{\infty} \rightarrow X_{\infty}$ of $X_{\infty}$ with center $V$, is defined in [13, §3] as the open formal subscheme of $X_{\infty}^{\prime}$ where $t$ generates $\mathcal{I O}_{X_{\infty}^{\prime}}$. It has the following universal property: $Y_{\infty}$ is $R$-flat, and $h_{s}: Y_{s} \rightarrow X_{s}$ factors through $V \rightarrow X_{s}$. Moreover, for any morphism of stft formal $R$-schemes $h^{\prime}: Z_{\infty} \rightarrow X_{\infty}$, such that $Z_{\infty}$ is $R$-flat and $h_{s}^{\prime}$ factors through $V$, there exists a unique morphism $v: Z_{\infty} \rightarrow Y_{\infty}$ with $h^{\prime}=h \circ v$. In particular, for any finite unramified extension $R^{\prime}$ of $R$, and for any point $\psi$ in $X_{\infty}\left(R^{\prime}\right)$ with $\psi(0) \in V$, the unique point of $X_{\infty}^{\prime}\left(R^{\prime}\right)$ that maps to $\psi$ is contained in $Y_{\infty}\left(R^{\prime}\right)$.

\subsection{Normal crossings and resolution of singularities.}

Definition 2.1. Let $X$ be a regular flat $R$-scheme (resp. a regular flat stft formal $R$-scheme). We say $X_{s}$ is a strict normal crossing divisor, if we can find, for each closed point $x$ of $X_{s}$, a regular system of local parameters $\left(x_{0}, \ldots, x_{m}\right)$ in the local ring $\mathcal{O}_{X, x}$, such that $t=u \prod_{i=0}^{m} x_{i}^{M_{i}}$, with $u$ a unit, and $M_{i} \in \mathbb{N}$.

Let $X$ be a regular flat $R$-variety (resp. a regular flat stft formal $R$-scheme) whose special fiber is a strict normal crossing divisor. Let $E_{i}, i \in I$, be the irreducible components of $\left(X_{s}\right)_{\text {red }}$. The variety $E_{i}$ is smooth over $k$, for each $i \in I$. We'll denote by $N_{i}$ the length of the local ring of $X_{s}$ at the generic point of $E_{i}$, and we call $N_{i}$ the multiplicity of $E_{i}$ in $X_{s}$. We write $X_{s}=\sum_{i \in I} N_{i} E_{i}$. We say that 
$X_{s}$ is a tame strict normal crossing divisor, if each $N_{i}$ is prime to the characteristic exponent $p$ of the residue field $k$.

For any non-empty subset $J$ of $I$, we define $E_{J}:=\cap_{i \in J} E_{i}$ and $E_{J}^{o}:=E_{J} \backslash$ $\left(\cup_{i \notin J} E_{i}\right)$. Moreover, we put $m_{J}:=\operatorname{gcd}\left\{N_{i} \mid i \in J\right\}$.

Definition 2.2. A resolution of singularities of a generically smooth flat $R$-variety $X$ (resp. a generically smooth, flat stft formal $R$-scheme), is a proper birational morphism of flat $R$-varieties (resp. a morphism of flat stft formal $R$-schemes) $h: X^{\prime} \rightarrow X$, such that $h$ induces an isomorphism on the generic fibers, and such that $X^{\prime}$ is regular, with as special fiber a strict normal crossing divisor $X_{s}^{\prime}=\sum_{i \in I} N_{i} E_{i}$. We say that the resolution $h$ is tame if $X_{s}^{\prime}$ is a tame strict normal crossing divisor.

Lemma 2.3. Let $A$ be an algebra topologically of finite type over $R$, and let $X$ be a scheme of finite type over $R$. If $\mathfrak{M}$ is a maximal ideal of $A$, defining a point $x$ of $\operatorname{Spf} A$, then the local morphism $A_{\mathfrak{M}} \rightarrow \mathcal{O}_{\mathrm{Spf} A, x}$ induces an isomorphism on the completions (w.r.t. the respective maximal ideals)

$$
\widehat{A}_{\mathfrak{M}} \cong \widehat{\mathcal{O}}_{\text {Spf } A, x}
$$

If $x$ is a point on the special fiber $X_{s}$ of $X$, then the local morphism $\mathcal{O}_{X, x} \rightarrow \mathcal{O}_{\widehat{X}, x}$ induces a canonical isomorphism on the completions

$$
\widehat{\mathcal{O}}_{X, x} \cong \widehat{\mathcal{O}}_{\widehat{X}, x}
$$

Proof. The first point is shown in the proof of [16, 1.2.1]. As for the second, note that for any $n \geq 0$, and with $X_{n}:=X \times_{R}\left(R / t^{n+1}\right)$,

$$
\left(\mathcal{O}_{X, x}\right) /\left(t^{n+1}\right) \cong \mathcal{O}_{X_{n}, x} \cong\left(\mathcal{O}_{\widehat{X}, x}\right) /\left(t^{n+1}\right)
$$

Lemma 2.4. Let $A$ be an algebra topologically of finite type over $R$, and let $X$ be a separated scheme of finite type over $R$.

(1) Spec $A$ is regular iff $\operatorname{Spf} A$ is regular. Moreover, $X$ is regular iff $\widehat{X}$ is regular.

(2) $(\operatorname{Spec} A)_{s}$ is a strict normal crossing divisor iff $(\operatorname{Spf} A)_{s}$ is a strict normal crossing divisor. Moreover, $X_{s}$ is a strict normal crossing divisor iff $\widehat{X}_{s}$ is a strict normal crossing divisor.

(3) Spf $A$ is generically smooth, iff $A \otimes_{R} K$ is geometrically regular over $K$. Moreover, if $X$ is generically smooth, then $\widehat{X}$ is generically smooth.

(4) if $K$ is perfect, any regular stft formal $R$-scheme is generically smooth.

Proof. Regularity of a local Noetherian ring is equivalent to regularity of its completion [27, 17.1.5], so (1) follows from Lemma 2.3. Point (2) follows from the fact that, for any local Noetherian ring $S$, a tuple $\left(x_{0}, \ldots, x_{m}\right)$ in $S$ is a regular system of local parameters for $S$ iff it is a regular system of local parameters for $\widehat{S}$.

So let us prove (3). Smoothness of the affinoid space $\mathcal{Y}:=\operatorname{Sp}\left(A \otimes_{R} K\right)$ over $K$ is equivalent to geometric regularity of $\mathcal{O}_{\mathcal{Y}, x}$ over $K$ for each point $x$ of $\mathcal{Y}$, by [12, 2.8]. If we denote again by $x$ the corresponding closed point of $Y:=\operatorname{Spec}\left(A \otimes_{R} K\right)$, then the natural local morphism of local Noetherian rings $\mathcal{O}_{Y, x} \rightarrow \mathcal{O}_{\mathcal{Y}, x}$ induces an isomorphism on the completions

$$
\widehat{\mathcal{O}}_{\mathcal{Y}, x} \cong \widehat{\mathcal{O}}_{Y, x}
$$

by $[10,5.2 .6 / 1,7.3 .2 / 3,7.3 .2 / 7]$. So the statement follows from the fact that a local Noetherian ring is geometrically regular over $K$, iff its completion is (by [27, 17.1.5] 
and the fact that, for any finite field extension $K^{\prime} / K$ and any local Noetherian ring $C$ over $K, \widehat{C} \otimes_{K} K^{\prime} \cong C \widehat{\otimes_{K} K^{\prime}}$.

If $X$ is generically smooth, then so is $\widehat{X}$, since by [8, 0.3.5], the generic fiber $X_{\eta}$ of $\widehat{X}$ is an open rigid subspace of the analytification of $X \times_{R} K$, which is smooth by [16, A.2.1].

Finally, to establish (4), it suffices to note that for any affinoid algebra $B$, regularity of $B$ implies geometric regularity of $B$ over $K$. This follows from 27 19.6.4-5].

Proposition 2.5. If $k$ has characteristic zero, any affine generically smooth flat stft formal $R$-scheme $X_{\infty}=\operatorname{Spf} A$ admits a resolution of singularities by means of formal admissible blow-ups with smooth centers.

Proof. First, we show that the scheme $X=\operatorname{Spec} A$ admits a resolution of singularities $h: X^{\prime} \rightarrow X$ by means of blow-ups with smooth centers, concentrated in the special fiber $X_{s}$. By [14, 8.2], it suffices to prove that, for any $n \geq 1$, the algebra $T_{n}:=R\left\{x_{1}, \ldots, x_{n}\right\}$ of convergent power series over $R$ satisfies the following properties:

(1) $\operatorname{Der}_{k}\left(T_{n}\right)$ is a finite projective $\left(T_{n}\right)$-module, locally of rank $(n+1)$,

(2) if $\mathfrak{M}$ is a maximal ideal of $T_{n}$, then the dimension of the localization of $T_{n}$ at $\mathfrak{M}$ equals $n+1$, and the residue field $T_{n} / \mathfrak{M}$ is algebraic over $k$.

Point (1) follows from the fact that $\operatorname{Der}_{k}\left(T_{n}\right) \cong\left(T_{n}\right)^{n+1}$, since a $k$-derivation on $T_{n}$ is determined by the images of $t$ and the $x_{i}$ (such a derivation $D$ is automatically continuous, since $D\left(t^{i}\right)$ is contained in the ideal $\left(t^{i-1}\right)$ by the Leibniz rule). Point (2) follows from the fact that the completion of this localization is isomorphic to $\widehat{\mathcal{O}}_{\mathbb{A}_{R}^{n}, x}$ for some closed point $x$ on $\mathbb{A}_{R}^{n}$, by Lemma 2.3 .

By [9, 2.6.6] and Lemma 2.4 the $t$-adic completion of $h$ is a resolution of singularities $h: X_{\infty}^{\prime} \rightarrow X_{\infty}$ by means of formal admissible blow-ups with smooth centers.

2.5. Étale cohomology of rigid varieties. Berkovich developed an étale cohomology theory for non-archimedean analytic spaces (including the rigid spaces) in 6. Throughout this paper, all cohomology will be Berkovich' étale cohomology, unless explicitly stated otherwise. We fix a prime $\ell$, invertible in $k$. We define, for any analytic space $\mathscr{Y}$ over the completion of some algebraic extension of $K$,

$$
\begin{aligned}
& H^{*}\left(\mathscr{Y}, \mathbb{Z}_{\ell}\right)=\lim ^{*} H^{*}\left(\mathscr{Y}, \mathbb{Z} / \ell^{n}\right) \\
& H^{*}\left(\mathscr{Y}, \mathbb{Q}_{\ell}\right)=H^{*}\left(\mathscr{Y}, \mathbb{Z}_{\ell}\right) \otimes \mathbb{Q}_{\ell} .
\end{aligned}
$$

We will simply write $H^{*}(\mathscr{Y})$ for $H^{*}\left(\mathscr{Y}, \mathbb{Q}_{\ell}\right)$, and we'll denote the graded vector space $\oplus_{i} H^{i}(\mathscr{Y})$ by $H(\mathscr{Y})$.

\section{Motivic Serre invariants with SUpport}

3.1. The Grothendieck ring of varieties. Let $Z$ be a variety over $k$. Consider the free Abelian group, generated by the isomorphism classes $[X]$ of $Z$-varieties $X$. We take the quotient of this group w.r.t. the following relations: whenever $X$ is a $Z$-variety, and $Y$ is a closed subvariety of $X$, we impose $[X]=[X \backslash Y]+[Y]$. This quotient is called the Grothendieck group of varieties over $Z$, and is denoted by $K_{0}\left(\operatorname{Var}_{Z}\right)$. We denote the class $\left[\mathbb{A}_{Z}^{1}\right]$ of the affine line over $Z$ by $\mathbb{L}_{Z}$, or by $\mathbb{L}$ if there is no risk of confusion. 
A constructible subset $C$ of a $Z$-variety $X$ can be written as a disjoint union of locally closed subsets, and defines unambiguously an element $[C]$ of $K_{0}\left(\operatorname{Var}_{Z}\right)$. When $Z$ is a separated scheme of finite type over $k$, we will write $K_{0}\left(\operatorname{Var}_{Z}\right)$ instead of $K_{0}\left(\operatorname{Var}_{Z_{\text {red }}}\right)$. For any separated scheme $X$ of finite type over $Z$, we will write $[X]$ instead of $\left[X_{\text {red }}\right]$.

We can define a product on $K_{0}\left(\operatorname{Var}_{Z}\right)$ as follows: for any pair of $Z$-varieties $X$, $Y$, we put $[X] .[Y]=\left[X \times_{Z} Y\right]$. This definition extends bilinearly to a product on $K_{0}\left(\operatorname{Var}_{Z}\right)$, and makes it into a ring, the Grothendieck ring of varieties over $Z$. The localized Grothendieck ring $\mathcal{M}_{Z}$ is obtained by inverting $\mathbb{L}_{Z}$ in $K_{0}\left(\operatorname{Var}_{Z}\right)$.

A morphism of $k$-varieties $f: W \rightarrow Z$ induces base-change ring morphisms $K_{0}\left(\operatorname{Var}_{Z}\right) \rightarrow K_{0}\left(\operatorname{Var}_{W}\right)$ and $\mathcal{M}_{Z} \rightarrow \mathcal{M}_{W}$, as well as forgetful morphisms of Abelian groups $K_{0}\left(\operatorname{Var}_{W}\right) \rightarrow K_{0}\left(\operatorname{Var}_{Z}\right)$ and $\mathcal{M}_{W} \rightarrow \mathcal{M}_{Z}$.

If $Z=\operatorname{Spec} k$, we write $K_{0}\left(\operatorname{Var}_{k}\right), \mathcal{M}_{k}$, and $\mathbb{L}$, rather than $K_{0}\left(\operatorname{Var}_{\mathrm{Spec} k}\right)$, $\mathcal{M}_{\text {Spec } k}$, and $\mathbb{L}_{\text {Spec } k}$.

The Grothendieck group $K_{0}\left(\operatorname{Var}_{Z}\right)$ is a universal additive invariant for $Z$-varieties: if $A$ is an Abelian group, and $\chi$ is an invariant of $Z$-varieties taking values in $A$, such that, for any $Z$-variety $X$ and any closed subvariety $Y \subset X, \chi(X)=\chi(X \backslash Y)+$ $\chi(Y)$, then $\chi$ factors uniquely through a group morphism $\chi: K_{0}\left(\operatorname{Var}_{Z}\right) \rightarrow A$, defined by $\chi([X])=\chi(X)$. If $A$ is a ring, and $\chi$ is multiplicative, i.e. $\chi\left(\left(X \times{ }_{Z} Y\right)_{\text {red }}\right)=$ $\chi(X) \cdot \chi(Y)$ for any pair of $Z$-varieties $X, Y$, then $\chi: K_{0}\left(\operatorname{Var}_{Z}\right) \rightarrow A$ is a morphism of rings.

For instance, for any $k$-variety $X$, we can consider its topological Euler characteristic $\chi_{\text {top }}(X)$. Fix a prime $\ell$, invertible in $k$. Then $\chi_{\text {top }}(X)$ is defined as

$$
\chi_{\text {top }}(X):=\sum_{i \geq 0}(-1)^{i} \operatorname{dim} H_{c}^{i}\left(X \times_{k} k^{s}, \mathbb{Q}_{\ell}\right)
$$

where $H_{c}^{i}\left(., \mathbb{Q}_{\ell}\right)$ is $\ell$-adic étale cohomology with proper support, and $k^{s}$ is a separable closure of $k$. This is an additive invariant, hence defines a morphism of groups

$$
\chi_{\text {top }}: K_{0}\left(\operatorname{Var}_{Z}\right) \rightarrow \mathbb{Z}
$$

for any $k$-variety $Z$. It is multiplicative for $Z=\operatorname{Spec} k$, so we get a morphism of rings

$$
\chi_{\text {top }}: K_{0}\left(\operatorname{Var}_{k}\right) \rightarrow \mathbb{Z}
$$

3.2. Motivic Serre invariants with support. Let $X_{\eta}$ be a separated, quasicompact smooth rigid $K$-variety. A weak Néron $R$-model for $X_{\eta}$ is a smooth stft formal scheme $U_{\infty}$ over $\operatorname{Spf} R$, whose generic fiber is an open rigid subspace of $X_{\eta}$, and which has the property that the natural map $U_{\infty}\left(R^{s h}\right) \rightarrow X_{\eta}\left(K^{s h}\right)$ is bijective [13. Definition 1.3]. By this latter property, we mean that $U_{\infty}\left(R^{\prime}\right) \rightarrow X_{\eta}\left(K^{\prime}\right)$ is bijective, for any finite unramified extension $K^{\prime}$ of $K$, where $R^{\prime}$ is the normalization of $R$ in $K^{\prime}$. Observe that this map is always injective, since $U_{\infty}$ is separated.

Definition 3.1. Let $h: U_{\infty} \rightarrow X_{\infty}$ be a morphism of stft flat formal $R$-schemes, with $X_{\eta}$ smooth over $K$. We say that $h$ is a Néron $R$-smoothening for $X_{\infty}$, if it has the following properties:

(1) $U_{\infty}$ is a weak Néron $R$-model for $X_{\eta}$,

(2) there exists a morphism $X_{\infty}^{\prime} \rightarrow X_{\infty}$ of stft flat formal $R$-schemes, inducing an isomorphism $X_{\eta}^{\prime} \rightarrow X_{\eta}$ on the generic fibers, such that $h$ factors through an open immersion $U_{\infty} \rightarrow X_{\infty}^{\prime}$. 
The result in [13, $\S 3$, Theorem 3.1] can be interpreted in our context as follows :

Theorem 3.2. If $X_{\infty}$ is a generically smooth, stft flat formal $R$-scheme, then $X_{\infty}$ admits a Néron $R$-smoothening. Moreover, we can always find an admissible blow-up $X_{\infty}^{\prime} \rightarrow X_{\infty}$, such that $\operatorname{Sm}\left(X_{\infty}^{\prime}\right) \rightarrow X_{\infty}$ is a Néron $R$-smoothening.

In [39], we refined the notion of motivic Serre invariant, first introduced in [34, as follows:

Definition 3.3 (Motivic Serre invariant). If $X_{\infty}$ is a generically smooth, stft flat formal $R$-scheme, the (relative) motivic Serre invariant $S\left(X_{\infty}\right)$ of $X_{\infty}$, is the class $\left[U_{s}\right]$ in $K_{0}\left(\operatorname{Var}_{X_{s}}\right) /\left(\mathbb{L}_{X_{s}}-\left[X_{s}\right]\right)$, where $U_{\infty} \rightarrow X_{\infty}$ is any Néron R-smoothening of $X_{\infty}$.

We proved in 39, Theorem 6.1, that this definition does not depend on the choice of the Néron $R$-smoothening, in the case where $X_{\eta}$ has pure dimension. We proved the general case in 38, Theorem 5.9.

If $Y_{\infty} \rightarrow X_{\infty}$ is a morphism of generically smooth, stft flat formal $R$-schemes, inducing an isomorphism on the generic fibers, the forgetful morphism

$$
K_{0}\left(\operatorname{Var}_{Y_{s}}\right) /\left(\mathbb{L}_{Y_{s}}-\left[Y_{s}\right]\right) \rightarrow K_{0}\left(\operatorname{Var}_{X_{s}}\right) /\left(\mathbb{L}_{X_{s}}-\left[X_{s}\right]\right)
$$

maps $S\left(Y_{\infty}\right)$ to $S\left(X_{\infty}\right)$. Hence, the motivic Serre invariants computed over all stft flat formal $R$-models of a separated, quasi-compact, smooth rigid variety $X_{\eta}$ over $K$, form a projective system.

For any stft flat formal $R$-model $X_{\infty}$ of $X_{\eta}$, the Serre invariant $S\left(X_{\eta}\right)$ defined in [34], is the image of $S\left(X_{\infty}\right)$ under the forgetful morphism

$$
K_{0}\left(\operatorname{Var}_{X_{s}}\right) /\left(\mathbb{L}_{X_{s}}-\left[X_{s}\right]\right) \rightarrow K_{0}\left(\operatorname{Var}_{k}\right) /(\mathbb{L}-1)
$$

It only depends on the rigid space $X_{\eta}$, and not on the choice of the formal model $X_{\infty}$

Definition 3.4. For any locally closed subscheme $V$ of $X_{s}$, we define the motivic Serre invariant $S_{V}\left(X_{\infty}\right)$ of $X_{\infty}$ with support in $V$ as the image of $S\left(X_{\infty}\right)$ under the base change morphism

$$
K_{0}\left(\operatorname{Var}_{X_{s}}\right) /\left(\mathbb{L}_{X_{s}}-\left[X_{s}\right]\right) \rightarrow K_{0}\left(\operatorname{Var}_{V}\right) /\left(\mathbb{L}_{V}-[V]\right)
$$

We will think of $S_{V}\left(X_{\infty}\right)$ as a measure for the number of unramified points on the tube $] V[$.

In general, this tube is not quasi-compact, so we cannot take its motivic Serre invariant in a direct way.

Proposition 3.5. (1) If $h: U_{\infty} \rightarrow X_{\infty}$ is a Néron $R$-smoothening, then

$$
S_{V}\left(X_{\infty}\right)=\left[U_{s} \times_{X_{s}} V\right] \in K_{0}\left(\operatorname{Var}_{V}\right) /\left(\mathbb{L}_{V}-[V]\right)
$$

(2) If $Y_{\infty}$ is any open formal subscheme of $X_{\infty}$ containing $V$, then $S_{V}\left(X_{\infty}\right)=$ $S_{V}\left(Y_{\infty}\right)$. In particular, if $V$ is open in $X_{s}$, then $S_{V}\left(X_{\infty}\right)=S\left(\widehat{X_{\infty} / V}\right)$.

(3) Suppose that $V$ is closed in $X_{s}$, and denote by $\pi: Y_{\infty} \rightarrow X_{\infty}$ the dilatation with center $V$. Then $S_{V}\left(X_{\infty}\right)$ is the image of $S\left(Y_{\infty}\right)$ under the forgetful morphism

$$
K_{0}\left(\operatorname{Var}_{Y_{s}}\right) /\left(\mathbb{L}_{Y_{s}}-\left[Y_{s}\right]\right) \rightarrow K_{0}\left(\operatorname{Var}_{V}\right) /\left(\mathbb{L}_{V}-[V]\right)
$$


JOHANNES NICAISE AND JULIEN SEBAG

Proof. Point (1) is clear. Point (2) follows from the fact that, if $h: U_{\infty} \rightarrow X_{\infty}$ is a Néron $R$-smoothening, then $h: h^{-1}\left(Y_{\infty}\right) \rightarrow Y_{\infty}$ is one, as well.

Finally, as for (3), let $B l_{V}: X_{\infty}^{\prime} \rightarrow X_{\infty}$ be the formal blow-up of of $X_{\infty}$ at $V$. The dilatation $Y_{\infty}$ is an open formal subscheme of $X_{\infty}^{\prime}$. Take a Néron $R$ smoothening $h: U_{\infty} \rightarrow X_{\infty}^{\prime}$. If we denote the complement of $Y_{s}$ in $B l_{V}^{-1}(V)$ by $E$, it suffices to prove that $\left(U_{\infty} \backslash h^{-1}(E)\right) \rightarrow X_{\infty}^{\prime}$ is still a Néron $R$-smoothening. This follows from the fact that the tube $] E\left[\right.$ in $X_{\infty}^{\prime}$ does not contain any $K^{\prime}$-points, with $K^{\prime}$ finite and unramified over $K$, by the universal property of the dilatation. Computing $S_{V}\left(X_{\infty}\right)$ on this Néron smoothening, we get

$$
S_{V}\left(X_{\infty}\right)=\left[h^{-1}\left(Y_{s}\right)\right]=S\left(Y_{\infty}\right)
$$

in $K_{0}\left(\operatorname{Var}_{V}\right) /\left(\mathbb{L}_{V}-[V]\right)$.

\section{WEAK NÉRON MODELS FOR RAMIFICATIONS}

Let $X_{\infty}$ be a regular flat stft formal $R$-scheme, such that its special fiber $X_{s}$ is a strict normal crossing divisor $\sum_{i \in I} N_{i} E_{i}$. We fix an integer $d>0$, prime to $p$. The aim of this section, is to construct a Néron $R$-smoothening for the ramification $X(d)_{\infty}:=X_{\infty} \times{ }_{R} R(d)$.

Choose any subset $J$ of $I$, such that $m_{J}$ is prime to $p$. We can cover $E_{J}^{o}$ by affine open formal subschemes $U_{\infty}=\operatorname{Spf} V$, such that on $U_{\infty}$, we can write $t=u \prod_{i \in J} x_{i}^{N_{i}}$, with $u$ a unit. We define an étale cover $U_{\infty}^{\prime} \rightarrow U_{\infty}$ by $U_{\infty}^{\prime}=$ Spf $V\{T\} /\left(u T^{m_{J}}-1\right)$. These covers glue together, and we obtain an étale cover $\widetilde{E}_{J}^{o} \rightarrow E_{J}^{o}$.

Remark. If $k$ has characteristic zero, and $X_{\infty}$ is isomorphic to the formal completion of $Z \times \times_{k[t]} k[[t]]$, for some smooth irreducible $k$-variety $Z$, and some dominant morphism $Z \rightarrow \operatorname{Spec} k[t]$, then our cover $\widetilde{E}_{J}^{o} / E_{J}^{o}$ coincides with the one defined in [24, 2.3].

We denote by $\widetilde{X(d)_{\infty}} \rightarrow X(d)_{\infty}$ the normalization of $X(d)_{\infty}$ (see [16] on normalization of formal schemes), and we denote by $\widetilde{E(d)}{ }_{i}^{o}$ the inverse image $\widetilde{X(d)}{ }_{s} \times_{X_{s}} E_{i}^{o}$ of $E_{i}^{o}$ in $\widetilde{X(d)}$, for each $i \in I$.

Definition 4.1. Let $J$ be a non-empty subset of $I$. We say that an integer $d \geq 1$ is $J$-linear if there exists, for each $j \in J$, an integer $\alpha_{j} \in \mathbb{N}^{*}$, such that

$$
d=\sum_{j \in J} \alpha_{j} N_{j}
$$

We say that an integer $d \geq 1$ is $X_{\text {s }}$-linear if there exists a subset $J \subset I$, with $|J|>1$ and $E_{J}^{o} \neq \emptyset$, such that $d$ is $J$-linear.

We recall two Lemmas from [39.

Lemma 4.2 (39, Lemma 5.15). Let $R^{\prime}$ be a finite extension of $R$ of ramification degree $d$, and consider an element $\psi$ of $X_{\infty}\left(R^{\prime}\right)$. If $J$ is the unique subset of I with $\psi(0) \in E_{J}^{o}$, then $d$ is J-linear.

Lemma 4.3 (39], Lemma 5.17). There exists a sequence of admissible blow-ups $\pi^{(j)}: X_{\infty}^{(j+1)} \rightarrow X_{\infty}^{(j)}, j=0, \ldots, r-1$, such that

- $X_{\infty}^{(0)}=X_{\infty}$, 
MOTIVIC SERRE INVARIANTS, RAMIFICATION, AND THE ANALYTIC MILNOR FIBER11

- the special fiber of $X_{\infty}^{(j)}$ is a strict normal crossing divisor

$$
X_{s}^{(j)}=\sum_{i \in I^{(j)}} N_{i}^{(j)} E_{i}^{(j)},
$$

- $\pi^{(j)}$ is the formal blow-up with center $E_{J^{(j)}}^{(j)}$, for some subset $J^{(j)}$ of $I^{(j)}$, with $\left|J^{(j)}\right|>1$,

- $d$ is not $X_{s}^{(r)}$-linear.

Lemma 4.4. If $d$ is not $X_{s}$-linear, then

$$
\operatorname{Sm}\left(\widetilde{X(d)_{s}}\right)=\bigsqcup_{N_{i} \mid d} \widetilde{E(d)_{i}^{o}}
$$

Moreover, for any $i \in I$ with $N_{i} \mid d$, the morphism $\widetilde{X(d)_{\infty}} \rightarrow \widetilde{X\left(N_{i}\right)_{\infty}}$ induces an isomorphism

$$
\widetilde{E(d)}_{i}^{o} \cong{\widetilde{E\left(N_{i}\right)_{i}}}^{o}
$$

and the $E_{i}^{o}$-variety $\widetilde{E\left(N_{i}\right)_{i}}$ is canonically isomorphic to $\widetilde{E}_{i}^{o}$.

Proof. Let $x$ be a smooth point of $\widetilde{X(d)}$. Since $\widetilde{X(d)_{\infty}}$ is flat, this implies that $\widetilde{X(d)_{\infty}} \rightarrow \operatorname{Spf} R(d)$ is smooth at $x$, and hence, there exists a section $\psi: \operatorname{Spf} R^{\prime} \rightarrow$ $\widehat{X(d)_{\infty}}$, with $R^{\prime}$ an unramified extension of $R(d)$, and $\psi(0)=x$. If we denote by $J$ the unique subset of $I$ such that the image of $x$ in $X_{s}$ belongs to $E_{J}^{o}$, then Lemma 4.2. combined with the hypothesis that $d$ is not $X_{s}$-linear, implies that $J$ is a singleton $\{i\}$, and $N_{i} \mid d$. Hence,

$$
\operatorname{Sm}\left(\widetilde{X(d)_{s}}\right) \subset \bigsqcup_{N_{i} \mid d} \widetilde{E(d)_{i}^{o}}
$$

Conversely, it follows from 31] (proof of the semi-stable reduction theorem II, pages 198-202) that

$$
\left.\bigsqcup_{N_{i} \mid d} \widetilde{E(d)}_{i}^{o} \subset S m(\widetilde{X(d)})_{s}\right)
$$

(their arguments in the algebraic setting carry over to formal schemes).

In fact, we can give an explicit description of $\operatorname{Sm}\left(\widetilde{\left.X(d)_{\infty}\right)}\right.$. Choose $i \in I$ such that $N_{i} \mid d$, and let $x$ be any closed point of $E_{i}^{o}$. Choose an affine open formal neighborhood $U_{\infty}=\operatorname{Spf} V$ of $x$ in $X_{\infty}$, such that on $U_{\infty}$, we can write $t=u x_{i}^{N_{i}}$, with $u$ a unit. For any minimal prime ideal $\mathfrak{P}$ of $V$, we can write

$$
\left(\frac{x_{i}}{\left(t_{d}\right)^{d / N_{i}}}\right)^{N_{i}}-u^{-1}=0
$$

in the quotient field of $V / \mathfrak{P}$, so the normalization map $\widetilde{U(d)_{\infty}} \rightarrow U(d)_{\infty}$ factors through

$$
\widetilde{U(d)_{\infty}} \rightarrow Y(d):=\operatorname{Spf} V(d)\{T\} /\left(\left(t_{d}\right)^{d / N_{i}} T-x_{i}, u T^{N_{i}}-1\right) \rightarrow U(d)_{\infty}
$$

where $V(d):=V \otimes_{R} R(d)$. We'll show that $Y(d)$ is smooth over $R(d)$. In particular, $Y(d)$ is normal, so $\widetilde{U(d)_{\infty}} \rightarrow Y(d)$ is an isomorphism. 
Since $Y(d)$ is flat over $R(d)$, it suffices to show that the special fiber $Y(d)_{s}$ is smooth over $k$. Reduction modulo $t_{d}$ yields

$$
Y(d)_{s}=\operatorname{Spec} V[T] /\left(x_{i}, u T^{N_{i}}-1\right)
$$

The section $x_{i}$ is part of a regular system of local parameters, so the scheme Spec $V /\left(x_{i}\right)$ is regular. Since $k$ is perfect, Spec $V /\left(x_{i}\right)$ is smooth over $k$. But $Y(d)_{s}$ is étale over Spec $V /\left(x_{i}\right)$, and hence smooth over $k$.

Finally, the explicit description $\widetilde{U(d)_{\infty}} \cong \operatorname{Spf} V(d)\{T\} /\left(\left(t_{d}\right)^{d / N_{i}} T-x_{i}, u T^{N_{i}}-1\right)$ shows that $\widetilde{E(d)_{i}}$ does not depend on $d$, as long as $N_{i} \mid d$. In fact, the restriction of ${\widetilde{E(d)_{i}}}_{i}^{o}$ over $U_{s}$ is given explicitly by $\operatorname{Spec} V[T] /\left(x_{i}, u T^{N_{i}}-1\right)$, which is canonically isomorphic to the restriction of $\widetilde{E}_{i}^{o}$ over $U_{s}$.

Theorem 4.5. Let $X_{\infty}$ be a regular, generically smooth, flat stft formal $R$-scheme, such that its special fiber $X_{s}$ is a strict normal crossing divisor $\sum_{i \in I} N_{i} E_{i}$. Let $d>0$ be an integer, prime to $p$, such that $d$ is not $X_{s}$-linear. Then

$$
\operatorname{Sm}\left(\widetilde{X(d)_{\infty}}\right) \rightarrow X(d)_{\infty}
$$

is an Néron $R$-smoothening, and

$$
S\left(X(d)_{\infty}\right)=\sum_{N_{i} \mid d}\left[\widetilde{E}_{i}^{o}\right]
$$

in $K_{0}\left(\operatorname{Var}_{X_{s}}\right) /\left(\mathbb{L}_{X_{s}}-\left[X_{s}\right]\right)$.

Proof. We only have to prove that $\operatorname{Sm}\left(\widetilde{X(d)_{\infty}}\right)$ is a weak Néron model for $X(d)_{\eta}$. This follows from Lemma 4.2, and Lemma 4.4 .

\section{A trace fORMUla FOR NON-ARChimedeAn ANALYTiC SPACES}

The purpose of this section is to prove a Grothendieck trace formula for nonarchimedean analytic spaces.

We suppose that the residue field $k$ is algebraically closed. Let $\varphi$ be a topological generator of the tame Galois group $G\left(K^{t} / K\right)$.

When $\mathscr{Y}$ is a rigid space over an algebraic, tamely ramified extension $K^{\prime}$ of $K$, we denote by $\overline{\mathscr{Y}}$ the base change $\mathscr{Y} \widehat{\times}_{K^{\prime}} \widehat{K^{t}}$. Fix a prime $\ell$, different from $p$.

Lemma 5.1. Let $F$ be a separably closed field, and let $G=<g>$ be a finite cyclic group. Let $Y$ be a normal proper $F$-variety, and let $U$ be an open subscheme of $Y$. Let $\mathcal{L}$ be a lisse constructible $\overline{\mathbb{Q}}_{\ell}$-sheaf on $U$, tamely ramified on $Y-U$. Suppose that $G$ acts on $\mathcal{L}$. For any closed point $x$ on $U$,

$$
\operatorname{Tr}\left(g \mid H_{c}(U, \mathcal{L})\right)=\chi_{\text {top }}(U) \operatorname{Tr}\left(g \mid \mathcal{L}_{x}\right) .
$$

Proof. By definition, $\mathcal{L}$ is a lisse constructible sheaf over some finite extension $Q$ of $\mathbb{Q}_{\ell}$. The tamely ramified lisse sheaf $\mathcal{L}$ on $U$ with stalk $\mathcal{L}_{x}$ at $x$ is determined by a continuous morphism

$$
\psi: \pi_{1}^{t}(U, x) \rightarrow \operatorname{Aut}_{Q}\left(\mathcal{L}_{x}\right) .
$$

Giving an endomorphism $f$ of $\mathcal{L}$, amounts to giving an element $\rho(f)$ of $\operatorname{End}_{Q}\left(\mathcal{L}_{x}\right)$, such that $\rho(f) \psi(\gamma)=\psi(\gamma) \rho(f)$, for each $\gamma \in \pi_{1}^{t}(U, x)$.

Passing to a finite extension of $Q$, we can decompose $\mathcal{L}$ as $\oplus_{i} \mathcal{L}_{i}$, according to the decomposition of $\mathcal{L}_{x} \otimes \overline{\mathbb{Q}}_{\ell}$ into generalized eigenspaces w.r.t. $\rho(g)$. Hence, we may as well assume that $\rho(g)$ has only one eigenvalue $e$ on $\mathcal{L}_{x} \otimes \overline{\mathbb{Q}}_{\ell}$. 
MOTIVIC SERRE INVARIANTS, RAMIFICATION, AND THE ANALYTIC MILNOR FIBER13

Write the automorphism induced by $\rho(g)$ on $\mathcal{L}_{x}$ as e.Id $+N$, with $N$ nilpotent. Since $\operatorname{Tr}\left(N \mid H_{c}(U, \mathcal{L})\right)=0$, it suffices to prove that

$$
\text { e. } \chi(U, \mathcal{L})=\operatorname{Tr}\left(e . I d \mid H_{c}(U, \mathcal{L})\right)=\chi(U, Q) \operatorname{Tr}\left(e . I d \mid \mathcal{L}_{x}\right)=\chi_{\text {top }}(U) \cdot \operatorname{e.rank}\left(\mathcal{L}_{x}\right) .
$$

This follows from [30, Cor 2.7.

Lemma 5.2. Let $Y$ be a regular flat variety over $R$, such that $Y_{s}$ is a strict normal crossing divisor $\sum_{i \in I} N_{i} E_{i}$. If $J$ is a subset of $I$, with $|J|>1$, and $B l_{E_{J}}$ is the blow-up of $Y$ at $E_{J}$, with exceptional component $E_{0}^{\prime}$, then $\left(E_{0}^{\prime}\right)^{o}$ is a Zariski-locally trivial fibration over $E_{J}^{o}$, and its fiber is a torus $\mathbb{G}_{m, k}^{|J|-1}$. In particular, $\left[\left(E_{0}^{\prime}\right)^{o}\right]=$ $\left[E_{J}^{o}\right]\left(\mathbb{L}_{X_{s}}-\left[X_{s}\right]\right)^{|J|-1}$ in $K_{0}\left(\operatorname{Var}_{X_{s}}\right)$.

Proof. Let $x$ be any closed point on $E_{J}$, and take a regular system of local parameters $\left(x_{0}, \ldots, x_{m}\right)$ on $Y$ at $x$, such that $t=u \prod_{i \in J} x_{i}^{N_{i}}$, with $u$ a unit, where we identified $J$ with a subset of $\{0, \ldots, m\}$. The sequence $\left(x_{i}\right)_{i \in J}$ is regular in a neighborhood $U$ of $x$. Now apply [25, IV-26], to the blow-up of $U$ with center $\left(x_{i}\right)_{i \in J}$.

Lemma 5.3. Let $Y$ be a regular flat variety over $R$, such that $Y_{s}$ is a tame strict normal crossing divisor $\sum_{i \in I} N_{i} E_{i}$. The complex of $\ell$-adic tame nearby cycles $R \psi_{\eta}^{t}\left(\mathbb{Q}_{\ell}\right)$ is constructible and tamely ramified on $Y_{s}$, and lisse on the strata $E_{J}^{o}$. For any closed point $y$ of $Y_{s}$, and for any integer $d>0$ prime to $p$,

$$
\begin{aligned}
& \operatorname{Tr}\left(\varphi^{d} \mid R \psi_{\eta}^{t}\left(\mathbb{Q}_{\ell}\right)_{y}\right)=N_{i} \quad \text { if } y \in E_{i}^{o} \text { with } N_{i} \mid d, \\
& \operatorname{Tr}\left(\varphi^{d} \mid R \psi_{\eta}^{t}\left(\mathbb{Q}_{\ell}\right)_{y}\right)=0 \quad \text { else. }
\end{aligned}
$$

Proof. This follows from the explicit computation of the nearby cycles in [1, I,3.3].

Theorem 5.4 (Trace formula). Let $X$ be a generically smooth flat variety over $R$, and suppose that $X$ admits a tame resolution $h: Y \rightarrow X$, with $Y_{s}=\sum_{i \in I} N_{i} E_{i}$. Let $Z$ be a proper subvariety of $X_{s}$. For any integer $d>0$, prime to $p$, we have

$$
\chi_{t o p}\left(S_{Z}(\widehat{X(d)})\right)=\operatorname{Tr}\left(\varphi^{d} \mid H(\overline{] Z[})\right)=\sum_{N_{i} \mid d} N_{i} \chi_{t o p}\left(h^{-1}(Z) \cap E_{i}^{o}\right) .
$$

Proof. We may suppose that $X=Y$.

By Berkovich' quasi-isomorphism [7, 3.5],

$$
\operatorname{Tr}\left(\varphi^{d} \mid H(\overline{Z[})\right)=\operatorname{Tr}\left(\varphi^{d} \mid H\left(Z, R \psi_{\eta}^{t}\left(\mathbb{Q}_{\ell}\right)\right)\right) .
$$

By Lemma 5.1, and Lemma 5.3 .

$$
\operatorname{Tr}\left(\varphi^{d} \mid H(\overline{] Z[})\right)=\sum_{N_{i} \mid d} N_{i} \chi_{t o p}\left(Z \cap E_{i}^{o}\right),
$$

We use Lemma 4.3 to construct a resolution $Y^{\prime} \rightarrow X$, with $Y_{s}^{\prime}=\sum_{i \in I^{\prime}} N_{i}^{\prime} E_{i}^{\prime}$, such that $d$ is not $Y_{s}^{\prime}$-linear. Denote by $Z^{\prime}$ the inverse image of $Z$ in $Y_{s}^{\prime}$.

By Theorem 4.5,

$$
\chi_{t o p}\left(S_{Z}(\widehat{X(d)})\right)=\sum_{N_{i}^{\prime} \mid d} \chi_{t o p}\left(\widetilde{Z}_{i}\right)
$$

where $\widetilde{Z}_{i}$ denotes the inverse image of $Z^{\prime} \cap\left(E_{i}^{\prime}\right)^{o}$ in $\widetilde{E_{i}^{\prime}}$. Since $\widetilde{E_{i}^{\prime}}$ is étale over $\left(E_{i}^{\prime}\right)^{o}$, of degree $N_{i}^{\prime}$, and tamely ramified, $\chi_{\text {top }}\left(\widetilde{Z}_{i}\right)=N_{i}^{\prime} \chi_{\text {top }}\left(Z^{\prime} \cap\left(E_{i}^{\prime}\right)^{o}\right)$, for any $i$. 
When $E_{i}^{\prime}$ is an exceptional component of $Y^{\prime} \rightarrow Y$, then $\chi_{\text {top }}\left(Z^{\prime} \cap\left(E_{i}^{\prime}\right)^{o}\right)=0$, by Lemma 5.2. Hence,

$$
\chi_{t o p}\left(S_{Z}(\widehat{X(d)})\right)=\sum_{N_{i} \mid d} N_{i} \chi_{t o p}\left(Z \cap E_{i}^{o}\right)
$$

The conditions of Theorem 5.4 are satisfied, in particular, when $k$ has characteristic zero.

Corollary 5.5. Suppose that $k$ is an algebraically closed field of characteristic zero. If $X_{K}^{a n}$ is the analytification of a smooth, proper $K$-variety $X_{K}$, then

$$
\chi_{\text {top }}\left(S\left(X_{K}^{a n} \times_{K} K(d)\right)\right)=\operatorname{Tr}\left(\varphi^{d} \mid H\left(X_{K} \times_{K} K^{s}, \mathbb{Q}_{\ell}\right)\right)
$$

for any integer $d>0$.

Proof. If $X$ is any flat $R$-model for $X_{K}$, then $X_{K}^{a n}$ is canonically isomorphic to the generic fiber $X_{\eta}$ of $\widehat{X}$, by [8, 0.3.5]. Moreover, by [6, 7.5.4], there is a canonical isomorphism

$$
H^{i}\left(X_{K} \times_{K} K^{s}, \mathbb{Q}_{\ell}\right) \cong H^{i}\left(\overline{X_{\eta}}\right)
$$

for each $i \geq 0$.

Remark. That some tameness condition is needed in the statement of the Trace Formula, is already clear from the following example: let $R$ be the ring $W\left(\mathbb{F}_{p}^{s}\right)$ of Witt vectors over $\mathbb{F}_{p}^{s}$, where $p>0$ is a prime. Let $X_{\infty}$ be the formal $R$-scheme $\operatorname{Spf} R\{x\} /\left(x^{p}-p\right)$. Obviously, $S\left(X_{\eta}\right)=0$, while $\operatorname{Tr}\left(\varphi \mid H\left(X_{\eta} \widehat{\times}_{K} \widehat{K}^{t}\right)\right)=1$, when $\varphi$ is a topological generator of the tame Galois group $G\left(K^{t} / K\right)$.

It should be possible to replace $X$ in the statement of the trace formula by an arbitrary generically smooth stft formal $R$-scheme $X_{\infty}$ which admits a tame resolution of singularities. Also the condition that $Z$ is proper does not seem essential. In particular, we expect that

$$
\chi_{\text {top }}\left(S\left(X_{\eta} \times_{K} K(d)\right)\right)=\operatorname{Tr}\left(\varphi^{d} \mid H\left(\overline{X_{\eta}}\right)\right)
$$

holds for any separated smooth quasi-compact rigid variety $X_{\eta}$ over $K$, if $k$ has characteristic zero (and also for any separated smooth rigid variety $X_{\eta}$ over $K$ which can be realized as a tube in a stft formal $R$-scheme, using the motivic Serre invariant with support in the left hand side).

\section{ORDER OF A GAUGE FORM}

We assume $R=k[[t]]$, with $k$ a field of characteristic zero. Let $X_{\infty}$ be a regular, flat stft formal $R$-scheme, of pure relative dimension $m$, such that the special fiber $X_{s}$ is a strict normal crossing divisor $\sum_{i \in I} N_{i} E_{i}$. Let $\omega$ be a gauge form on the generic fiber $X_{\eta}$. The purpose of this section, is to define the order $\operatorname{ord}_{E_{i}} \omega$ of $\omega$ at the generic point of $E_{i}$, for any $i \in I$. 
6.1. Motivic integral of a gauge form on a smooth rigid variety. If $X_{\infty}$ is smooth, this order was defined already in [13, 4.3], for general $R$. This definition was used in [34, 4.3.1] to give an expression for the integral of a gauge form on a separated, smooth, quasi-compact rigid variety over $K$. In [39, we refined this notion as follows. Let $Y_{\infty}$ be a generically smooth, flat stft formal $R$-scheme, of pure relative dimension $m$, and let $\omega$ be a gauge form on $Y_{\eta}$. We take a Néron $R$ smoothening $h: Z_{\infty} \rightarrow Y_{\infty}$, and we denote by $\mathcal{C}$ the set of connected components of $Z_{s}$. Then

$$
\int_{Y_{\infty}}|\omega|:=\mathbb{L}^{-m} \sum_{U \in \mathcal{C}}[U] \mathbb{L}^{-o r d_{U}\left(h^{*} \omega\right)} \in \mathcal{M}_{Y_{s}}
$$

depends only on the pair $\left(Y_{\infty}, \omega\right)$, and not on $Z_{\infty}$ (see [39], Lemma 6.4). The image of $\int_{Y_{\infty}}|\omega|$ under the forgetful morphism $\mathcal{M}_{Y_{s}} \rightarrow \mathcal{M}_{k}$ is the motivic integral $\int_{Y_{\eta}} \omega$ from [34, 4.1.2]. It depends only on the pair $\left(Y_{\eta}, \omega\right)$, and not on the model $Y_{\infty}$. Finally, the image of $\int_{Y_{\infty}}|\omega|$ under the projection morphism

$$
\mathcal{M}_{Y_{s}} \rightarrow \mathcal{M}_{Y_{s}} /\left(\mathbb{L}_{Y_{s}}-\left[Y_{s}\right]\right) \cong K_{0}\left(\operatorname{Var}_{Y_{s}}\right) /\left(\mathbb{L}_{Y_{s}}-\left[Y_{s}\right]\right)
$$

is exactly the motivic Serre invariant $S\left(Y_{\infty}\right)$ from Definition 3.3. In particular, it depends only on $Y_{\infty}$, and not on $\omega$.

6.2. The order of a top form at a section. First, we generalize a definition from [34, 4.1]. Let $Y_{\infty}$ be any flat st $f t$ formal $R$-scheme, equidimensional of relative dimension $m$. Let $R^{\prime}$ be a finite extension of $R$, of ramification index $e$.

Definition 6.1. For any element $\psi$ of $Y_{\infty}\left(R^{\prime}\right)$, and any ideal sheaf $\mathcal{I}$ on $Y_{\infty}$, we define $\operatorname{ord}(\mathcal{I})(\psi)$ as the length of the $R^{\prime}$-module $R^{\prime} / \psi^{*} \mathcal{I}$.

We recall that the length of the zero module is 0 , and the length of $R^{\prime}$ is $\infty$.

For any element $\psi$ of $Y_{\infty}\left(R^{\prime}\right)$, the $R^{\prime}$-module $M:=\left(\psi^{*} \Omega_{Y_{\infty} / R}^{m}\right) /($ torsion) is free of rank 1.

Definition 6.2. For any global section $\omega$ of $\Omega_{Y_{\infty} / R}^{m}$, we define the order of $\omega$ at $\psi$ as the length of the $R^{\prime}$-module $M / R^{\prime}\left(\psi^{*} \omega\right)$. We denote this value by $\operatorname{ord}(\omega)(\psi)$.

If $e=1$, this definition coincides with the one given in [34, 4.1]. It only depends on an open formal neighbourhood of $\psi(0)$ in $Y_{\infty}$.

If $\omega \in \Omega_{Y_{\eta} / K}^{m}\left(Y_{\eta}\right)$, there exists an integer $a \geq 0$ such that $t^{a} \omega \in \Omega_{Y_{\infty} / R}^{m}\left(Y_{\infty}\right)$, by the isomorphism of sheaves [12, 1.5]

$$
\Omega_{Y_{\eta} / K}^{m} \cong \Omega_{Y_{\infty} / R}^{m} \otimes_{R} K
$$

and the fact that $Y_{\infty}$ is quasi-compact.

Definition 6.3. If $\omega$ is a global section of $\Omega_{Y_{\eta} / K}^{m}$, we take an integer $a \geq 0$ such that $t^{a} \omega$ is defined on $Y_{\infty}$, and we define $\operatorname{ord}(\omega)(\psi)$ as $\operatorname{ord}\left(t^{a} \omega\right)(\psi)-e a$.

This definition does not depend on the choice of $a$. If $Y_{\eta}$ is smooth, and $\omega$ is a gauge form on $Y_{\eta}, \operatorname{ord}(\omega)(\psi)$ is finite.

Now let $h: Z_{\infty} \rightarrow Y_{\infty}$ be a morphism of flat, generically smooth stft formal $R$ schemes, both equidimensional of relative dimension $m$. Let $R^{\prime}$ be a finite extension of $R$, and fix a section $\psi$ in $Z_{\infty}\left(R^{\prime}\right)$. The canonical morphism

$$
h^{*} \Omega_{Y_{\infty} / R}^{m} \rightarrow \Omega_{Z_{\infty} / R}^{m}
$$


induces a morphism of free rank $1 R^{\prime}$-modules

$$
\left(\psi^{*} h^{*} \Omega_{Y_{\infty} / R}^{m}\right) /(\text { torsion }) \rightarrow\left(\psi^{*} \Omega_{Z_{\infty} / R}^{m}\right) /(\text { torsion })
$$

We define $\operatorname{ord}\left(J a c_{h}\right)(\psi)$ as the length of its cokernel.

If $\Omega_{Z_{\infty} / R}^{m} /$ (torsion) is a locally free rank 1 module over $\mathcal{O}_{Z_{\infty}}$, we define the Jacobian ideal sheaf $\mathcal{J} a c_{h}$ of $h$ as the annihilator of the cokernel of the morphism

$$
h^{*} \Omega_{Y_{\infty} / R}^{m} \rightarrow \Omega_{Z_{\infty} / R}^{m} /(\text { torsion })
$$

and we have $\operatorname{ord}\left(J a c_{h}\right)(\psi)=\operatorname{ord}\left(\mathcal{J} a c_{h}\right)(\psi)$. The following lemma generalizes 34, Lemma 4.1.1] :

Lemma 6.4. Let $h: Z_{\infty} \rightarrow Y_{\infty}$ be a morphism of flat, generically smooth stft formal $R$-schemes, both equidimensional of relative dimension $m$. Let $R^{\prime}$ be a finite extension of $R$. For any global section $\omega$ of $\Omega_{Y_{\eta} / K}^{m}$, and any $\psi^{\prime} \in Z_{\infty}\left(R^{\prime}\right)$,

$$
\operatorname{ord}\left(h^{*} \omega\right)(\psi)=\operatorname{ord}(\omega)(h(\psi))+\operatorname{ord}\left(J a c_{h}\right)(\psi)
$$

Proof. This follows immediately from the definitions.

Lemma 6.5. Suppose that $Y_{\infty}$ is a regular flat stft formal $R$-scheme, and that $Y_{s}$ is a strict normal crossing divisor. Let $\omega$ be a global section of $\Omega_{Y_{\eta} / K}^{m}$. We denote by $\widetilde{\omega(e)}$ the pullback of $\omega$ to the generic fiber of $\widetilde{Y(e)_{\infty}}$. Let $R^{\prime}$ be a finite extension of $R(e)$, and let $\psi(e)$ be a section in $\operatorname{Sm}\left(\widehat{\left.Y(e)_{\infty}\right)}\left(R^{\prime}\right)\right.$. If we denote by $\psi$ its image in $Y_{\infty}\left(R^{\prime}\right)$, then

$$
\operatorname{ord}(\omega)(\psi)=\operatorname{ord}(\widetilde{\omega(e)})(\psi(e))
$$

Proof. Consider the morphism

$$
h: S m\left(\widehat{Y(e)_{\infty}}\right) \rightarrow Y(e)_{\infty}
$$

Since $\Omega_{Y(e)_{\infty} / R(e)}^{m} \cong \Omega_{Y_{\infty} / R}^{m} \otimes_{R} R(e)$, it suffices to show, by Lemma 6.4, that the natural map

$$
\left(\psi(e)^{*} h^{*} \Omega_{Y(e)_{\infty} / R(e)}^{m}\right) /(\text { torsion }) \rightarrow\left(\psi(e)^{*} \Omega_{Y(e)_{\infty} / R(e)}^{m}\right) /(\text { torsion })
$$

is surjective. This follows from the explicit description of the normalization in the proof of Lemma 4.4 .

6.3. The order of a top form along a component of the special fiber. We recall that $X_{\infty}$ denotes a regular, flat stft formal $R$-scheme, of pure relative dimension $m$, such that the special fiber $X_{s}$ is a strict normal crossing divisor $\sum_{i \in I} N_{i} E_{i}$. Let $\omega$ be a gauge form on the generic fiber $X_{\eta}$. We denote by $\xi_{i}$ the generic point of $E_{i}$, for each $i$.

Lemma 6.6. The local ring $\mathcal{O}_{X_{\infty}, \xi_{i}}$ of $X_{\infty}$ at $\xi_{i}$, is a discrete valuation ring.

Proof. Locally at $\xi_{i}$, the divisor $E_{i}$ is defined by an equation $x_{0}=0$. The quotient $\mathcal{O}_{X_{\infty}, \xi_{i}} /\left(x_{0}\right)$ is isomorphic to $\mathcal{O}_{E_{i}, \xi_{i}}$, which is a field. Hence, $x_{0}$ generates the maximal ideal of $\mathcal{O}_{X_{\infty}, \xi_{i}}$, and since this ring is a noetherian integral domain, it is a discrete valuation ring.

Lemma 6.7. The $\left(\mathcal{O}_{X_{\infty}, \xi_{i}}\right)$-module $\Omega_{i}:=\left(\Omega_{X_{\infty} / R}^{m}\right)_{i} /($ torsion $)$ is free of rank one. 
Proof. Since $\left(\Omega_{X_{\infty} / R}^{m}\right)_{\xi_{i}}$ is finite over $\mathcal{O}_{X_{\infty}, \xi_{i}}$, and $\mathcal{O}_{X_{\infty}, \xi_{i}}$ is a PID, the module $\Omega_{i}$ is free over $\mathcal{O}_{X_{\infty}, \xi_{i}}$.

By [12, 1.5], we have

$$
\Omega_{X_{\infty} / R}^{m} \otimes_{R} K \cong \Omega_{X_{\eta} / K}^{m}
$$

and since $X_{\eta}$ is smooth, this is a rank 1 module over $\mathcal{O}_{X_{\eta}}$. Hence, $\Omega_{i}$ has rank 1 over $\mathcal{O}_{X_{\infty}, \xi}$.

Definition 6.8. If $\omega \in \Omega_{X_{\infty} / R}^{m}\left(X_{\infty}\right)$, we define the order of $\omega$ along $E_{i}$ as the length of the $\mathcal{O}_{X_{\infty}, \xi_{i}}$-module $\Omega_{i} /\left(\mathcal{O}_{X_{\infty}, \xi_{i}} \omega\right)$, and we denote it by $\operatorname{ord}_{E_{i}} \omega$.

If $\omega \in \Omega_{X_{\eta} / K}^{m}\left(X_{\eta}\right)$, there exists an integer $a \geq 0$ such that $t^{a} \omega \in \Omega_{X_{\infty} / R}^{m}\left(X_{\infty}\right)$, by the isomorphism of sheaves [12, 1.5]

$$
\Omega_{X_{\eta} / K}^{m} \cong \Omega_{X_{\infty} / R}^{m} \otimes_{R} K
$$

and the fact that $X_{\infty}$ is quasi-compact. We define the order of $\omega$ along $E_{i}$ as

$$
\operatorname{ord}_{E_{i}} \omega:=\operatorname{ord}_{E_{i}}\left(t^{a} \omega\right)-a N_{i}
$$

This definition does not depend on $a$. If $X_{\infty}$ is smooth, it coincides with the one in [13, 4.3]. It follows from Lemma 6.6 and Lemma 6.7 that $\operatorname{ord}_{E_{i}} \omega$ is finite if $\omega$ is a gauge form on $X_{\eta}$.

Lemma 6.9. Let $Y_{\infty}$ be a generically smooth stft formal $R$-scheme, endowed with an étale morphism of stft formal $R$-schemes $h: Y_{\infty} \rightarrow X_{\infty}$, such that the image of $h$ contains the point $\xi_{i}$. For any $\omega \in \Omega_{X_{\eta} / K}^{m}\left(X_{\eta}\right)$, and for any connected component $C$ of $h^{-1}\left(E_{i}\right)$, we have

$$
\operatorname{ord}_{E_{i}} \omega=\operatorname{ord}_{C} h^{*} \omega .
$$

Proof. Denote by $\xi^{\prime}$ the generic point of $C$. Since $h$ is étale, the local morphism $h^{*}$ : $\mathcal{O}_{X_{\infty}, \xi_{i}} \rightarrow \mathcal{O}_{Y_{\infty}, \xi^{\prime}}$ is a flat, unramified monomorphism, so we have isomorphisms of $\mathcal{O}_{Y_{\infty}, \xi^{\prime}}$-modules

$$
\begin{aligned}
\left(\Omega_{X_{\infty} / R}^{m}\right)_{\xi_{i}} \otimes_{\mathcal{O}_{X_{\infty}, \xi_{i}}} \mathcal{O}_{Y_{\infty}, \xi^{\prime}} & \cong\left(\Omega_{Y_{\infty} / R}^{m}\right)_{\xi^{\prime}} \\
\left(\Omega_{i}:=\left(\Omega_{X_{\infty} / R}^{m}\right)_{\xi_{i}} /(\text { torsion })\right) \otimes_{\mathcal{O}_{X_{\infty}, \xi_{i}}} \mathcal{O}_{Y_{\infty}, \xi^{\prime}} & \cong \Omega^{\prime}:=\left(\Omega_{Y_{\infty} / R}^{m}\right)_{\xi^{\prime}} /(\text { torsion }) \\
\left(\Omega_{i} /\left(\mathcal{O}_{X_{\infty}, \xi_{i}} \omega\right)\right) \otimes_{\mathcal{O}_{X_{\infty}, \xi_{i}}} \mathcal{O}_{Y_{\infty}, \xi^{\prime}} & \cong \Omega^{\prime} /\left(\mathcal{O}_{Y_{\infty}, \xi^{\prime}} h^{*} \omega\right)
\end{aligned}
$$

Now the result follows from the following algebraic property: if $g: A \rightarrow A^{\prime}$ is a flat, unramified morphism of discrete valuation rings, if $M$ is a free $A$-module of rank 1 , and $m$ is an element of $M$, then the length of the $A$-module $M /(A m)$ equals the length of the $A^{\prime}$-module $\left(M \otimes A^{\prime}\right) /\left(A^{\prime} m\right)$. Indeed: fixing an isomorphism of $A$-modules $A \cong M$, the length of $A /(A m)$ is equal to the valuation of $m$ in $A$.

We'll need the following technical lemma.

Lemma 6.10. Let $x$ be a closed point of a generically smooth, flat, stft formal $R$-scheme $Y_{\infty}$ of pure relative dimension $m$, and let $\omega$ be a global section of $\Omega_{Y_{\infty} / R}^{m}$ which induces a gauge form on $Y_{\eta}$. Let $\mathfrak{P}$ be a prime ideal of the completed local ring $\widehat{\mathcal{O}}_{Y_{\infty}, x}$ with $t \notin \mathfrak{P}$. If we denote by $\widehat{\mathcal{O}}_{\mathfrak{P}}$ the localization of $\widehat{\mathcal{O}}_{Y_{\infty}, x}$ at $\mathfrak{P}$, and if we put

$$
\widehat{\Omega}_{\mathfrak{P}}:=\left(\left(\Omega_{Y_{\infty} / R}^{m}\right)_{x} \otimes \widehat{\mathcal{O}}_{\mathfrak{P}}\right) /(\text { torsion })
$$

then $\omega \notin \mathfrak{P} \widehat{\Omega}_{\mathfrak{P}}$. 
Proof. We'll denote $\widehat{\mathcal{O}}_{Y_{\infty}, x}$ by $\widehat{\mathcal{O}}$. Note that

$$
\left(\Omega_{Y_{\infty} / R}^{m}\right)_{x} \otimes \widehat{\mathcal{O}} \cong \Omega_{\operatorname{Spf} \widehat{\mathcal{O}} / R}^{m}(\operatorname{Spf} \widehat{\mathcal{O}})=\Omega_{\widehat{\mathcal{O}} / R}^{m}
$$

Consider the tube $] x$ [ of $x$ in $X_{\infty}$; it is an open rigid subspace of $Y_{\eta}$, and coincides with the generic fiber of $\widehat{X_{\infty} / x}=\operatorname{Spf} \widehat{\mathcal{O}}$.

We put $C_{0}=\widehat{\mathcal{O}} \otimes_{R} K$ and $\Omega_{0}^{m}=\Omega_{\widehat{\mathcal{O}} / R}^{m} \otimes_{R} K$. By [17, 7.1.9], maximal ideals $M$ of $C_{0}$ are in canonical bijective correspondence with the points $z$ of $] x[$, and the completions of the respective local rings are isomorphic. Moreover, by [17, 7.1.12],

$$
\left(\Omega_{] x[/ K}^{m}\right)_{z} \otimes_{\mathcal{O}_{] x[, z}} \widehat{\mathcal{O}}_{] x[, z} \cong \Omega_{0}^{m} \otimes_{C_{0}} \widehat{\left(C_{0}\right)_{M}}
$$

Since $] x$ [ is smooth and $\omega$ is a gauge form, this implies that $\Omega_{0}^{m}$ is a free $C_{0}$-module, and $\omega$ is a generator.

Since $\mathfrak{P}$ does not contain $t, \mathfrak{Q}=\mathfrak{P} C_{0}$ is a prime ideal of $C_{0}$. Consider the flat ring morphism $i: \widehat{\mathcal{O}}_{\mathfrak{P}} \rightarrow\left(C_{0}\right)_{\mathfrak{Q}}$ and the map of $\widehat{\mathcal{O}}_{\mathfrak{P}}$-modules

$$
\left(\Omega_{Y_{\infty} / R}^{m}\right)_{x} \otimes \widehat{\mathcal{O}}_{\mathfrak{P}} \rightarrow \Omega_{0}^{m} \otimes\left(C_{0}\right)_{\mathfrak{Q}}
$$

Since $\Omega_{0}^{m}$ is free, and $i$ is flat, this map factors though

$$
\widehat{\Omega}_{\mathfrak{P}} \rightarrow \Omega_{0}^{m} \otimes\left(C_{0}\right)_{\mathfrak{Q}}
$$

If $\omega \in \mathfrak{P} \widehat{\Omega}_{\mathfrak{P}}$, then its image is contained in $\mathfrak{Q}\left(\Omega_{0}^{m} \otimes\left(C_{0}\right)_{\mathfrak{Q}}\right)$, which contradicts the fact that $\omega$ is a gauge form on $Y_{\eta}$.

For each $i \in I$, we denote by $\mathcal{I}_{E_{i}}$ the defining ideal sheaf of $E_{i}$ in $X_{\infty}$. For any finite extension $R^{\prime}$ of $R$ and any $\psi \in X_{\infty}\left(R^{\prime}\right)$, we denote $\operatorname{ord}\left(\mathcal{I}_{E_{i}}\right)(\psi)$ by $\operatorname{ord}_{E_{i}}(\psi)$ (see Definition 6.1). If $R^{\prime}$ has ramification degree $e$ over $R$, and the closed point $\psi(0)$ of the section $\psi$ is contained in $E_{i}^{o}$, then the equality $t=x_{i}^{N_{i}} *$ (unit) in $\mathcal{O}_{X_{\infty}, \psi(0)}$ implies that $\operatorname{ord}_{E_{i}}(\psi)=e / N_{i}$.

Lemma 6.11. Fix a non-empty subset $J$ of $I$, and an integer $e>0$. Let $R^{\prime}$ be a finite extension of $R$, of ramification index $e$, and let $\psi$ be an element of $X_{\infty}\left(R^{\prime}\right)$, such that its closed point $\psi(0)$ lies on $E_{J}^{o}$. For any gauge form $\omega$ on $X_{\eta}$,

$$
\operatorname{ord}(\omega)(\psi)=\sum_{i \in J} \operatorname{ord}_{E_{i}}(\psi)\left(\operatorname{ord}_{E_{i}} \omega-1\right)+\max _{i \in J}\left\{\operatorname{ord}_{E_{i}}(\psi)\right\}
$$

Proof. We may assume that $\omega \in \Omega_{X_{\infty} / R}^{m}\left(X_{\infty}\right)$. For notational reasons, we identify $J$ with a subset $\{0, \ldots, p\}$ of $\{0, \ldots, m\}$. We denote by $\xi_{j}$ the generic point of $E_{j}$, for $j \in J$, and by $\xi$ the generic point of $E_{J}$. We denote by $t^{\prime}$ a uniformizing parameter for $R^{\prime}$, with $\left(t^{\prime}\right)^{e}=t$.

By Lemma 6.9 and Lemma 6.4 we can pass to an étale cover and we may assume that we can find a regular system of local parameters $\left(x_{0}, \ldots, x_{m}\right)$ on $X_{\infty}$ at $x:=\psi(0)$, such that $t=\prod_{i \in J} x_{i}^{N_{i}}$.

We can write $\omega$ as

$$
\sum_{i=0}^{m} h_{i}\left(d x_{0} \wedge \ldots \wedge \widehat{d x}_{i} \wedge \ldots \wedge d x_{m}\right),
$$

with $h_{i} \in \mathcal{O}_{X_{\infty}, x}$ 
Fix $j \in J$. The equality $t=\prod_{i \in J} x_{i}^{N_{i}}$ implies that, for any $i \in J$,

$$
d x_{0} \wedge \ldots \wedge \widehat{d x}_{i} \wedge \ldots \wedge d x_{m}=(-1)^{j-i} \frac{N_{i} x_{j}}{N_{j} x_{i}} d x_{0} \wedge \ldots \wedge \widehat{d x_{j}} \wedge \ldots \wedge d x_{m}
$$

in $\left(\Omega_{X_{\infty} / R}^{m}\right)_{\xi_{j}} /($ torsion $)$, so this module is generated by

$$
d x_{0} \wedge \ldots \wedge \widehat{d x_{j}} \wedge \ldots \wedge d x_{m}
$$

and $\operatorname{ord}_{E_{j}} \omega$ equals the order of $\sum_{i \in J}(-1)^{i} h_{i} \frac{N_{i} x_{j}}{x_{i}}$ in the discrete valuation ring $\mathcal{O}_{X_{\infty}, \xi_{j}}$. Multiplying with the unit $\prod_{i \in J \backslash\{j\}} x_{i}$, we see that $\operatorname{ord}_{E_{j}} \omega$ equals the order of

$$
\alpha:=\sum_{i \in J}\left((-1)^{i} h_{i} N_{i} \prod_{\ell \in J \backslash\{i\}} x_{\ell}\right)
$$

in the discrete valuation ring $\mathcal{O}_{X_{\infty}, \xi_{j}}$, which is the same as the order $\operatorname{ord}_{x_{j}} \alpha$ of $\alpha$ w.r.t. $x_{j}$ in the unique factorization domain $\mathcal{O}_{X_{\infty}, x}$ (i.e. the largest integer $N$ such that $\left(x_{j}\right)^{N}$ divides $\alpha$ ).

A similar computation shows that

$$
\operatorname{ord}(\omega)(\psi)=\operatorname{ord}_{t^{\prime}} \sum_{i \in J}(-1)^{i} \frac{\psi^{*}\left(h_{i} x_{j}\right)}{\psi^{*}\left(x_{i}\right)} N_{i}
$$

where we chose $j \in J$ such that $\operatorname{ord}_{t^{\prime}} \psi^{*}\left(x_{j}\right)$ is the maximum of $\left\{\operatorname{ord}_{t^{\prime}} \psi^{*}\left(x_{i}\right) \mid i \in J\right\}$. Hence, it suffices to show that

$$
\operatorname{ord}_{t^{\prime}} \psi^{*} \alpha=\sum_{i \in J} \operatorname{ord}_{x_{i}} \alpha . \operatorname{ord}_{t^{\prime}} \psi^{*}\left(x_{i}\right)
$$

However, since $\omega$ is gauge on $X_{\eta}, \alpha$ is the product of a unit and a monomial in $\left(x_{i}\right)_{i \in J}$. Indeed: let $\mathfrak{P}$ be a prime ideal in the completed local ring $\widehat{\mathcal{O}}_{X_{\infty}, x}$ which does not contain $t$, and suppose that $\alpha \in \mathfrak{P}$. Denote by $\widehat{\mathcal{O}}_{\mathfrak{P}}$ the localization of $\widehat{\mathcal{O}}_{X_{\infty}, x}$ at $\mathfrak{P}$, and put

$$
\widehat{\Omega}_{\mathfrak{P}}:=\left(\left(\Omega_{X_{\infty} / R}^{m}\right)_{x} \otimes \widehat{\mathcal{O}}_{\mathfrak{P}}\right) /(\text { torsion })
$$

Since all the $x_{i}, i \in J$ are units in the local ring $\widehat{\mathcal{O}}_{\mathfrak{P}}$, we see by the same computation as above that $\alpha$ divides $\omega$ in $\widehat{\Omega}_{\mathfrak{P}}$, and hence $\omega \in \mathfrak{P} \widehat{\Omega}_{\mathfrak{P}}$, which is impossible by Lemma 6.10. So we may conclude that the only prime divisors of $\alpha$ in the unique factorization domain $\mathcal{O}_{X_{\infty}, x}$ are the prime divisors of $t$, i.e. the elements $x_{i}$, modulo multiplication with a unit.

The lemma now follows from the fact that $\operatorname{ord}_{t^{\prime}} \psi^{*} x_{i}=\operatorname{ord}_{E_{i}}(\psi)$ for each $i \in$ $J$.

Proposition 6.12. Let $X_{\infty}$ be a regular, flat stft formal $R$-scheme, such that the special fiber $X_{s}$ is a strict normal crossing divisor $\sum_{i \in I} N_{i} E_{i}$. Let $\omega$ be a gauge form on the generic fiber $X_{\eta}$. Take a subset $J$ of $I$, with $|J|>1$, and $E_{J}^{o} \neq \emptyset$. Let $h: X_{\infty}^{\prime} \rightarrow X_{\infty}$ be the formal blow-up with center $E_{J}$, and denote by $E_{0}^{\prime}$ its exceptional component. We have

$$
\operatorname{ord}_{E_{0}^{\prime}} \omega=\sum_{i \in J} \operatorname{ord}_{E_{i}} \omega
$$


Proof. Put $e=\sum_{i \in J} N_{i}$. We can find a finite unramified extension $R^{\prime}$ of $R(e)$, and a section $\psi^{\prime}$ in $X_{\infty}^{\prime}\left(R^{\prime}\right)$, such that the closed point of $\psi^{\prime}$ lies on $\left(E_{0}^{\prime}\right)^{o}$. Denote by $\psi$ the image of $\psi^{\prime}$ in $X_{\infty}\left(R^{\prime}\right)$. Note that $\operatorname{ord}_{E_{i}}(\psi)=1$ for each $i \in J$, since these orders are strictly positive, and $\sum_{i \in J} \operatorname{ord}_{E_{i}}(\psi) N_{i}$ equals $e$. Applying Lemma 6.11 to the section $\psi^{\prime}$, we get

$$
\operatorname{ord}(\omega)\left(\psi^{\prime}\right)=\operatorname{ord}_{E_{0}^{\prime}} \omega
$$

Applying Lemma 6.11 to the section $\psi$, we get

$$
\operatorname{ord}(\omega)(\psi)=\sum_{i \in J} \operatorname{ord}_{E_{i}} \omega-|J|+1
$$

At the generic point of $E_{0}^{\prime}$, the module $\Omega_{X_{\infty}^{\prime} / R}^{m} /$ (torsion) is free of rank 1 , and the Jacobian ideal sheaf of $h$ is the defining ideal sheaf of $(|J|-1) E_{0}^{\prime}$, so we may conclude by Lemma 6.4.

Proposition 6.13. Let $X_{\infty}$ be a regular, flat stft formal $R$-scheme, of pure relative dimension $m$, such that the special fiber $X_{s}$ is a strict normal crossing divisor $\sum_{i \in I} N_{i} E_{i}$. Let $\omega$ be a gauge form on the generic fiber $X_{\eta}$. Fix an integer $e>0$. Denote by $\widetilde{\omega(e)}$ the pullback of $\omega$ to the generic fiber of $\widetilde{X(e)_{\infty}}$. For each $i \in I$, with $N_{i} \mid e$, and each connected component $C$ of $\widetilde{E(e)_{i}}$, we have

$$
\operatorname{ord}_{C}(\widetilde{\omega(e)})=\left(e / N_{i}\right) . \operatorname{ord}_{E_{i}} \omega
$$

Proof. Fix $i \in I$, with $N_{i} \mid$ e. Since $\widehat{X_{\infty}(e)}$ is smooth along $C$, by Lemma 4.4, we can find an unramified finite extension $R^{\prime}$ of $R(e)$, and a section $\psi(e)$ in $\left.\widetilde{\left(X_{\infty}(e)\right.}\right)\left(R^{\prime}\right)$ such that its closed point lies on $C$. We denote by $\psi$ the image of $\psi(e)$ in $X_{\infty}\left(R^{\prime}\right)$. By Lemma 6.11,

$$
\operatorname{ord}(\omega)(\psi)=\left(e / N_{i}\right) \operatorname{ord}_{E_{i}} \omega \text { and } \operatorname{ord}(\widetilde{\omega(e)})(\psi(e))=\operatorname{ord}_{C}(\widetilde{\omega(e)})
$$

Now we can apply Lemma 6.5.

\section{Computation of the volume Poincaré series}

Throughout this section, we put $R=k[[t]]$, with $k$ a field of characteristic zero. Let $X_{\infty}$ be a generically smooth stft formal scheme over $R$, with generic fiber $X_{\eta}$. Let $\omega$ be a gauge form on $X_{\eta}$. For any integer $d>0$, we'll denote by $\omega(d)$ the pullback of $\omega$ to $X(d)_{\eta}$.

Definition 7.1. For any integer $d>0$, and any locally closed subset $Z$ of $X_{s}$, we put

$$
F\left(X_{\infty}, \omega ; d\right):=\int_{X(d)_{\infty}}|\omega(d)| \in \mathcal{M}_{X_{s}}
$$

and we define $F_{Z}\left(X_{\infty}, \omega ; d\right)$ as the image of $F\left(X_{\infty}, \omega ; d\right)$ under the base change morphism $\mathcal{M}_{X_{s}} \rightarrow \mathcal{M}_{Z}$. This defines functions

$$
F\left(X_{\infty}, \omega\right): \mathbb{N}^{*} \rightarrow \mathcal{M}_{X_{s}} \text { and } F_{Z}\left(X_{\infty}, \omega\right): \mathbb{N}^{*} \rightarrow \mathcal{M}_{Z}
$$

which we call the local singular series (resp. local singular series with support in $Z$ ) associated to the pair $\left(X_{\infty}, \omega\right)$.

Using terminology from [15, 4.4], we define the volume Poincaré series as a Mellin transform of the local singular series. 
MOTIVIC SERRE INVARIANTS, RAMIFICATION, AND THE ANALYTIC MILNOR FIBER21

Definition 7.2 (Volume Poincaré series). The volume Poincaré series $S\left(X_{\infty}, \omega ; T\right)$ of the pair $\left(X_{\infty}, \omega\right)$ is the generating series

$$
S\left(X_{\infty}, \omega ; T\right)=\sum_{d>0} F\left(X_{\infty}, \omega ; d\right) T^{d} \in \mathcal{M}_{X_{s}}[[T]]
$$

Its image in $\mathcal{M}_{k}[[T]]$ only depends on the pair $\left(X_{\eta}, \omega\right)$, and is given by

$$
S\left(X_{\eta}, \omega ; T\right)=\sum_{d>0}\left(\int_{X(d)_{\eta}}|\omega(d)|\right) T^{d}
$$

For any locally closed subset $Z$ of $X_{s}$, the volume Poincaré series $S_{Z}\left(X_{\infty}, \omega ; T\right)$ with support in $Z$ is defined as the image of $S\left(X_{\infty}, \omega ; T\right)$ under the base change morphism

$$
\mathcal{M}_{X_{s}}[[T]] \rightarrow \mathcal{M}_{Z}[[T]]
$$

Definition 7.3 (Serre Poincaré series). The Serre Poincaré series $S\left(X_{\infty} ; T\right)$ of $X_{\infty}$ is the generating series

$$
S\left(X_{\infty} ; T\right)=\sum_{d>0} S\left(X(d)_{\infty}\right) T^{d} \in K_{0}\left(\operatorname{Var}_{X_{s}}\right) /\left(\mathbb{L}_{X_{s}}-\left[X_{s}\right]\right)[[T]] .
$$

Its image in $K_{0}\left(\operatorname{Var}_{k}\right) /(\mathbb{L}-1)[[T]]$ only depends on $X_{\eta}$, and is given by

$$
S\left(X_{\eta} ; T\right)=\sum_{d>0} S\left(X(d)_{\eta}\right) T^{d} .
$$

For any locally closed subscheme $Z$ of $X_{s}$, the Serre Poincaré series with support in $Z$ is given by

$$
S_{Z}\left(X_{\infty} ; T\right)=\sum_{d>0} S_{Z}\left(X(d)_{\infty}\right) T^{d} \in K_{0}\left(\operatorname{Var}_{Z}\right) /\left(\mathbb{L}_{Z}-[Z]\right)[[T]] .
$$

The series $S\left(X_{\infty}, \omega ; T\right)$ specializes to the Serre Poincaré series $S\left(X_{\infty} ; T\right)$ under the morphism

$$
\mathcal{M}_{X_{s}} \rightarrow \mathcal{M}_{X_{s}} /\left(\mathbb{L}_{X_{s}}-\left[X_{s}\right]\right) \cong K_{0}\left(\operatorname{Var}_{X_{s}}\right) /\left(\mathbb{L}_{X_{s}}-\left[X_{s}\right]\right)
$$

Likewise, $S_{Z}\left(X_{\infty}, \omega ; T\right)$ specializes to $S_{Z}\left(X_{\infty} ; T\right)$

If $X_{\infty}$ is a regular, flat stft formal $R$-scheme, such that $X_{s}$ has strict normal crossings, and $\omega$ is a gauge form on $X_{\eta}$, we can give an explicit expression for the volume Poincaré series $S\left(X_{\infty}, \omega ; T\right)$. First, we need some technical lemmas.

Lemma 7.4. Let $F$ be any field, and consider a torus

$$
\mathbb{G}_{m, F}^{r}:=\operatorname{Spec} F\left[x_{1}, x_{1}^{-1}, \ldots, x_{r}, x_{r}^{-1}\right]
$$

Take $u \in F^{*}$, and integers $a, b_{1}, \ldots, b_{r}$ with $a>0$ and $\operatorname{gcd}\left(a, b_{1}, \ldots, b_{r}\right)=1$. Then the étale cover

$$
T:=\operatorname{Spec} F\left[x_{1}, x_{1}^{-1}, \ldots, x_{r}, x_{r}^{-1}\right][z] /\left(z^{a}-u \prod_{j=1}^{r} x_{j}^{b_{j}}\right)
$$

is isomorphic to $\mathbb{G}_{m, F}^{r}$ over $F$.

Proof. By Bézout, there exist integers $\alpha, \beta_{1}, \ldots, \beta_{r}$ with $\alpha . a+\sum_{j=1}^{r} \beta_{j} . b_{j}=1$. We can write $T$ as

$$
T \cong \operatorname{Spec} F\left[x_{1}, x_{1}^{-1}, \ldots, x_{r}, x_{r}^{-1}\right][z] /\left(\left(u^{-\alpha} z\right)^{a}-\prod_{j=1}^{r}\left(u^{\beta_{j}} x_{j}\right)^{b_{j}}\right)
$$


and hence, we may as well assume that $u=1$. In this case, writing

$$
\begin{gathered}
F\left[x_{1}, x_{1}^{-1}, \ldots, x_{r}, x_{r}^{-1}\right][z] /\left(z^{a}-\prod_{j=1}^{r} x_{j}^{b_{j}}\right) \\
\cong F\left[z, z^{-1}, x_{1}, x_{1}^{-1}, \ldots, x_{r}, x_{r}^{-1}\right] /\left(1-z^{-a} \prod_{j=1}^{r} x_{j}^{b_{j}}\right)
\end{gathered}
$$

we see that $T$ is isomorphic to the torus $\operatorname{Spec} F[L]$, where $L$ is the lattice

$$
\mathbb{Z}^{r+1} /\left(-a . e_{0}+\sum_{j=1}^{r} b_{j} \cdot e_{j}\right) \cong \mathbb{Z}^{r}
$$

$\left(e_{0}, \ldots, e_{r}\right.$ denotes the standard basis of $\left.\mathbb{Z}^{r+1}\right)$. Note that $L$ is torsion-free because $\operatorname{gcd}\left(a, b_{1}, \ldots, b_{r}\right)=1$.

Lemma 7.5. Let $X_{\infty}$ be a regular stft formal scheme over $R$, such that its special fiber $X_{s}=\sum_{i=1}^{q} N_{i} E_{i}$ is a strict normal crossing divisor. Let $J$ be a nonempty subset of $I=\{1, \ldots, q\}$, with $|J|>1$, and let $\pi_{X}: X_{\infty}^{\prime} \rightarrow X_{\infty}$ be the formal blow-up with center $E_{J}$. Denote its exceptional divisor by $E_{0}^{\prime}$, and the strict transform of $E_{i}$ by $E_{i}^{\prime}$, for $i \in I$. For each subset $K$ of $I$, the stratum $\left(\widetilde{E}^{\prime}\right)_{K \cup\{0\}}^{o}$ is a Zariski piecewisely trivial fibration over $\widetilde{E}_{J \cup K}^{o}$, and its fiber is a torus of dimension $|J \backslash K|-1$ (where we give the empty set dimension -1). This fibration is compatible with the structural morphisms to $X_{s}$. In particular, $\left[\left(\widetilde{E}^{\prime}\right)_{K \cup\{0\}}^{o}\right]=\left(\mathbb{L}_{X_{s}}-\left[X_{s}\right]\right)^{|J \backslash K|-1}\left[\widetilde{E}_{J \cup K}^{o}\right]$ in $K_{0}\left(\operatorname{Var}_{X_{s}}\right)$.

Proof. Fix a subset $K$ of $I$. Covering $E_{J \cup K}^{o} \subset X_{\infty}$ by opens $U$ in $X_{\infty}$, we may assume that

$$
t=u \prod_{j \in J \cup K} x_{j}^{N_{j}},
$$

with $u$ a unit, and with $x_{j}$ defining $E_{j}$. We put

$$
G:=\left(E^{\prime}\right)_{K \cup\{0\}}^{o} \times_{E_{J \cup K}^{o}} \widetilde{E}_{J \cup K}^{o}
$$

This means that $G$ is the étale cover of $\left(E^{\prime}\right)_{K \cup\{0\}}^{o}$ obtained by taking a $m_{J \cup K^{-}}$th root of $\pi_{X}^{*}(u)^{-1}$; we write this as

$$
G=\left(E^{\prime}\right)_{K \cup\{0\}}^{o}[w] /\left(\pi_{X}^{*}(u) w^{m_{J \cup K}}-1\right)
$$

We may assume that $1 \in J \backslash K$. We abbreviate $J \backslash\{1\}$ to $J^{-}$. Putting $x_{j}^{\prime}=x_{j}$ for $j \in(K \cup\{1\}) \backslash J^{-}$, and $x_{j}^{\prime}=x_{j} / x_{1}$, for $j \in J^{-}$, we can write

$$
t=\pi_{X}^{*}(u)\left(x_{1}^{\prime}\right)^{N_{0}} \prod_{j \in J^{-} \cup K}\left(x_{j}^{\prime}\right)^{N_{j}}
$$

on $X_{\infty}^{\prime} \backslash E_{1}^{\prime}$, with $N_{0}=\sum_{i \in J} N_{i}$. The locally closed subset $\left(E^{\prime}\right)_{K \cup\{0\}}^{o}$ is defined by $x_{j}^{\prime}=0$ for $j \in K \cup\{1\}$, and $x_{j}^{\prime} \neq 0$ for $j \in J^{-} \backslash K$.

We have an étale $\left(E^{\prime}\right)_{K \cup\{0\}}^{o}$-morphism

$$
\begin{array}{r}
\left(\widetilde{E}^{\prime}\right)_{K \cup\{0\}}^{o}=\left(E^{\prime}\right)_{K \cup\{0\}}^{o}[z] /\left(\pi_{X}^{*}(u) \prod_{j \in J^{-} \backslash K}\left(x_{j}^{\prime}\right)^{N_{j}} z^{m_{K \cup\{0\}}}-1\right) \\
\longrightarrow G=\left(E^{\prime}\right)_{K \cup\{0\}}^{o}[w] /\left(\pi_{X}^{*}(u) w^{m_{J \cup K}}-1\right),
\end{array}
$$

defined by

$$
w \mapsto z^{m_{K \cup\{0\}} / m_{J \cup K}} \prod_{j \in J^{-} \backslash K}\left(x_{j}^{\prime}\right)^{N_{j} / m_{J \cup K}}
$$


We see it is induced by the isomorphism

$$
\left(\widetilde{E}^{\prime}\right)_{K \cup\{0\}}^{o}=G[z] /\left(z^{m_{K \cup\{0\}} / m_{J \cup K}}-w \prod_{j \in J^{-} \backslash K}\left(x_{j}^{\prime}\right)^{-N_{j} / m_{J \cup K}}\right)
$$

We claim that the fiber $E_{x}$ of $\left(\widetilde{E}^{\prime}\right)_{K \cup\{0\}}^{o}$ over any point $x$ of $\widetilde{E}_{J \cup K}^{o}$ is isomorphic to $\mathbb{G}_{m, k(x)}^{|J \backslash K|-1}$. This concludes the proof, by [42], Theorem 4.2.3.

So let us prove our claim. Denote by $G_{x}$ the fiber of $G$ over $x$. The regular function $w$ is constant along $G_{x}$, with value $w(x) \in k(x)^{\times}$. By [25, IV-26], the restrictions of the functions $x_{j}^{\prime}, j \in J^{-} \backslash K$, to $G_{x}$, induce an isomorphism $G_{x} \cong$ Spec $k(x)\left[x_{j}^{\prime},\left(x_{j}^{\prime}\right)^{-1}\right]_{j \in J^{-} \backslash K}$ (if $A$ is an $R$-algebra topologically of finite type, the formal blow-up of $\operatorname{Spf} A$ at a regular center $\left(x_{j}\right)_{j \in J}$ contained in the special fiber, is the formal completion of the blow-up of Spec $A$ at the center $\left(x_{j}\right)_{j \in J}$; in particular, the special fibers are the same; so [25, IV-26] carries over to the formal case).

The fiber $E_{x}$ is isomorphic to

$$
G_{x}[z] /\left(z^{m_{K \cup\{0\}} / m_{J \cup K}}-w(x) \prod_{j \in J^{-} \backslash K}\left(x_{j}^{\prime}\right)^{-N_{j} / m_{J \cup K}}\right)
$$

We can conclude by Lemma 7.4 and the fact that

$$
\operatorname{gcd}\left(m_{K \cup\{0\}}, N_{j}\right)_{j \in J-\backslash K}=m_{J \cup K}
$$

Theorem 7.6. Let $X_{\infty}$ be a regular flat stft formal scheme over $R$, of pure relative dimension $m$, such that $X_{s}=\sum_{i \in I} N_{i} E_{i}$ is a strict normal crossing divisor. Let $\omega$ be a gauge form on $X_{\eta}$.

If we denote by $\mu_{i}$ the order ord $_{E_{i}} \omega$ of $\omega$ along $E_{i}$, then, for any integer $d>0$,

$$
F\left(X_{\infty}, \omega ; d\right)=\mathbb{L}^{-m} \sum_{\emptyset \neq J \subset I}(\mathbb{L}-1)^{|J|-1}\left[\widetilde{E}_{J}^{o}\right]\left(\sum_{\substack{k_{i} \geq 1, i \in J \\ \sum_{i \in J} k_{i} N_{i}=d}} \mathbb{L}^{-\sum_{i} k_{i} \mu_{i}}\right) \text { in } \mathcal{M}_{X_{s}}
$$

In particular,

$$
S\left(X(d)_{\infty}\right)=\sum_{i \in I, N_{i} \mid d}\left[\widetilde{E}_{i}^{o}\right] \text { in } K_{0}\left(\operatorname{Var}_{X_{s}}\right) /\left(\mathbb{L}_{X_{s}}-\left[X_{s}\right]\right)
$$

Proof. First, suppose that $d$ is not $X_{s}$-linear. By Theorem $4.5, \operatorname{Sm}\left(\widetilde{X(d)_{\infty}}\right) \rightarrow$ $X(d)_{\infty}$ is a Néron smoothening. By Lemma 6.13, for each $i \in I$ with $N_{i} \mid d$, the order of $\widetilde{\omega(d)}$ along any component of $\widetilde{E(d)}_{i}^{o}$ equals $d \mu_{i} / N_{i}$.

By 34, Prop. 4.3.1 and [39], Lemma 6.4, we see that

$$
\begin{aligned}
F\left(X_{\infty}, \omega ; d\right) & =\mathbb{L}^{-m} \sum_{N_{i} \mid d}\left[\widetilde{E}_{i}^{o}\right] \mathbb{L}^{-d \mu_{i} / N_{i}} \\
& =\mathbb{L}^{-m} \sum_{\emptyset \neq J \subset I}(\mathbb{L}-1)^{|J|-1}\left[\widetilde{E}_{J}^{o}\right]\left(\sum_{\substack{k_{i} \geq 1, i \in J \\
\sum_{i \in J} k_{i} N_{i}=d}} \mathbb{L}^{-\sum_{i} k_{i} \mu_{i}}\right),
\end{aligned}
$$

provided that $d$ is not $X_{s}$-linear.

We use Lemma 7.5 to show that the expression $\left(^{*}\right)$ does not change under the formal blow-up $h$ of a stratum $E_{J}$. Denote the exceptional divisor of $h$ by $E_{0}^{\prime}$, and the strict transform of $E_{i}$ by $E_{i}^{\prime}$, for $i \in I$. 
Observe that the multiplicity $N_{0}$ of $E_{0}^{\prime}$ in $X_{s}^{\prime}$ equals $\sum_{j \in J} N_{j}$, and that, by Lemma 6.12, $\mu_{0}:=\operatorname{ord}_{E_{0}^{\prime}} \omega=\sum_{j \in J} \mu_{j}$. For any non-empty subset $L$ of $I$, containing $J$, and any vector $k=\left(k_{i}\right)$ in $\left(\mathbb{N}^{*}\right)^{L}$, denote by $M(k)$ the set of indices $j \in J$ such that $k_{j}=m(k):=\min \left\{k_{i} \mid i \in J\right\}$. We get, for each subset $L$ of $I$ containing $J$,

$$
\begin{aligned}
& (\mathbb{L}-1)^{|L|-1}\left[\widetilde{E}_{L}^{o}\right]\left(\sum_{\substack{k_{i} \geq 1, i \in L \\
\sum_{i \in L} k_{i} N_{i}=d}} \mathbb{L}^{-\sum_{i} k_{i} \mu_{i}}\right) \\
& =\sum_{L \backslash J \subset K \subset L}(\mathbb{L}-1)^{|L|-1}\left[\widetilde{E}_{L}^{o}\right]\left(\sum_{\substack{k=\left(k_{i}\right) \in\left(\mathbb{N}^{*}\right)^{L}, M(k)=L \backslash K \\
\sum_{i \in L} k_{i} N_{i}=d}} \mathbb{L}^{-\sum_{i} k_{i} \mu_{i}}\right) \\
& =\sum_{L \backslash J \subset K \subset L}(\mathbb{L}-1)^{|K|}\left[\left(\widetilde{E}^{\prime}\right)_{K \cup\{0\}}^{o}\right] \\
& \left(\sum_{\substack{k=\left(k_{i}\right) \in\left(\mathbb{N}^{*}\right)^{L} \\
M(k)=L \backslash K, \gamma(k)}} \mathbb{L}^{-m(k)\left(\sum_{i \in J} \mu_{i}\right)-\sum_{i \in J}\left(k_{i}-m(k)\right) \mu_{i}-\sum_{i \in L \backslash J} k_{i} \mu_{i}}\right)
\end{aligned}
$$

where condition $\gamma(k)$ means

$$
m(k)\left(\sum_{i \in J} N_{i}\right)+\sum_{i \in J}\left(k_{i}-m(k)\right) N_{i}+\sum_{i \in L \backslash J} k_{i} N_{i}=d .
$$

We can rewrite this last expression as

$$
\sum_{K \subset L, J \cup K=L}(\mathbb{L}-1)^{|K|}\left[\left(\widetilde{E}^{\prime}\right)_{K \cup\{0\}}^{o}\right]\left(\sum_{\substack{k^{\prime}=\left(k_{i}^{\prime}\right) \in\left(\mathbb{N}^{*}\right)^{K \cup\{0\}} \\ \sum_{i} k_{i}^{\prime} N_{i}=d}} \mathbb{L}^{-\sum_{i} k_{i}^{\prime} \mu_{i}}\right) .
$$

By Lemma 4.3. we can find, for each $d>0$, a composition of blow-ups $\pi^{(j)}$ : $X_{\infty}^{(j+1)} \rightarrow X_{\infty}^{(j)}, j=0, \ldots, r-1$, such that

- $X_{\infty}^{(0)}=X_{\infty}$

- the special fiber of $X_{\infty}^{(j)}$ is a strict normal crossing divisor

$$
X_{s}^{(j)}=\sum_{i=1}^{k_{j}} N_{i}^{(j)} E_{i}^{(j)}
$$

- $\pi^{(j)}$ is a blow-up with center $E_{J^{(j)}}$, for some non-empty subset $J^{(j)}$ of $\left\{1, \ldots, k_{j}\right\}$

- $d$ is not $X_{s}^{(r)}$-linear.

Since the expression $(*)$ does not change under any of the $\pi^{(j)}$, it is valid for all $d$.

Corollary 7.7. Let $X_{\infty}$ be a generically smooth stft formal $R$-scheme, of pure relative dimension $m$. Suppose that $X_{\infty}$ admits a resolution $X_{\infty}^{\prime} \rightarrow X_{\infty}$, with special fiber $X_{s}^{\prime}=\sum_{i \in I} N_{i} E_{i}$. Let $\omega$ be a gauge form on $X_{\eta}$.

The volume Poincaré series $S\left(X_{\infty}, \omega ; T\right)$ is rational over $\mathcal{M}_{X_{s}}$. In fact, if we put $\mu_{i}:=\operatorname{ord}_{E_{i}} \omega$, then the series is given explicitly by

$$
S\left(X_{\infty}, \omega ; T\right)=\mathbb{L}^{-m} \sum_{\emptyset \neq J \subset I}(\mathbb{L}-1)^{|J|-1}\left[\widetilde{E}_{J}^{o}\right] \prod_{i \in J} \frac{\mathbb{L}^{-\mu_{i}} T^{N_{i}}}{1-\mathbb{L}^{-\mu_{i}} T^{N_{i}}} \text { in } \mathcal{M}_{X_{s}}[[T]]
$$


MOTIVIC SERRE INVARIANTS, RAMIFICATION, AND THE ANALYTIC MILNOR FIBER 25

In particular,

$$
S\left(X_{\infty} ; T\right)=\sum_{i \in I}\left[\widetilde{E}_{i}^{o}\right] \frac{T^{N_{i}}}{1-T^{N_{i}}} \in\left(K_{0}\left(\operatorname{Var}_{X_{s}}\right) /\left(\mathbb{L}_{X_{s}}-\left[X_{s}\right]\right)\right)[[T]]
$$

Proof. We may assume that $X_{\infty}^{\prime}=X_{\infty}$. By Theorem 7.6 .

$$
\begin{aligned}
& S\left(X_{\infty}, \omega ; T\right)=\mathbb{L}^{-m} \sum_{d>0} \sum_{\emptyset \neq J \subset I}\left((\mathbb{L}-1)^{|J|-1}\left[\widetilde{E}_{J}^{o}\right]\left(\sum_{\substack{k_{i} \geq 1, i \in J \\
\sum_{i \in J} k_{i} N_{i}=d}} \mathbb{L}^{-\sum_{i} k_{i} \mu_{i}}\right)\right) T^{d} \\
& =\mathbb{L}^{-m} \sum_{\emptyset \neq J \subset I}\left((\mathbb{L}-1)^{|J|-1}\left[\widetilde{E}_{J}^{o}\right] \prod_{i \in J} \sum_{k_{i}>0} \mathbb{L}^{-k_{i} \mu_{i}} T^{k_{i} N_{i}}\right) \\
& =\mathbb{L}^{-m} \sum_{\emptyset \neq J \subset I}\left((\mathbb{L}-1)^{|J|-1}\left[\widetilde{E}_{J}^{o}\right] \prod_{i \in J} \frac{\mathbb{L}^{-\mu_{i}} T^{N_{i}}}{1-\mathbb{L}^{-\mu_{i}} T^{N_{i}}}\right)
\end{aligned}
$$

By Proposition 2.5. any affine generically smooth flat stft formal $R$-scheme admits a resolution of singularities. By the additivity of the motivic integral, we obtain an expression for the volume Poincaré series in terms of a finite atlas of local resolutions. In particular, we obtain the following result.

Corollary 7.8. Let $X_{\infty}$ be a generically smooth stft formal $R$-scheme, of pure relative dimension $m$. Let $\omega$ be a gauge form on $X_{\eta}$. The volume Poincaré series $S\left(X_{\infty}, \omega ; T\right)$ is rational over $\mathcal{M}_{X_{s}}$. More precisely, there exists a finite subset $S$ of $\mathbb{Z} \times \mathbb{N}^{*}$ such that $S\left(X_{\infty}, \omega ; T\right)$ belongs to the subring

$$
\mathcal{M}_{X_{s}}\left[\frac{\mathbb{L}^{a} T^{b}}{1-\mathbb{L}^{a} T^{b}}\right]_{(a, b) \in S}
$$

of $\mathcal{M}_{X_{s}}[[T]]$

\section{The motivic Volume}

Suppose that $R=k[[t]]$, with $k$ a field of characteristic zero.

Let $X_{\infty}$ be a generically smooth, flat stft formal $R$-scheme, of pure relative dimension $m$, and let $\omega$ be a gauge form on $X_{\eta}$. It is not possible to associate a motivic Serre invariant to $X_{\infty} \widehat{\times} \widehat{R^{s}}$ in a direct way, since the normalization $R^{s}$ of $R$ in $K^{s}$ is not a discrete valuation ring. We will define a motivic object by taking a limit of Serre invariants of finite ramifications of $X_{\infty}$, instead.

Definition $8.1(28],(2.8))$. There is a unique $\mathcal{M}_{X_{s}}$-linear morphism

$$
\lim _{T \rightarrow \infty}: \mathcal{M}_{X_{s}}\left[\frac{\mathbb{L}^{a} T^{b}}{1-\mathbb{L}^{a} T^{b}}\right]_{(a, b) \in \mathbb{Z} \times \mathbb{N}^{*}} \longrightarrow \mathcal{M}_{X_{s}}
$$

mapping

$$
\prod_{(a, b) \in I} \frac{\mathbb{L}^{a} T^{b}}{1-\mathbb{L}^{a} T^{b}}
$$

to $(-1)^{|I|}=(-1)^{|I|}\left[X_{s}\right]$, for each finite subset $I$ of $\mathbb{Z} \times \mathbb{N}^{*}$. We call the image of an element its limit for $T \rightarrow \infty$. 
To see that this morphism is well-defined, note that the image of an element is given by the constant term of its Taylor development in $T^{-1}$.

Proposition 8.2. The limit of $-S\left(X_{\infty}, \omega ; T\right)$ for $T \rightarrow \infty$ is well-defined, and does not depend on the choice of $\omega$. If $X_{\infty}^{\prime} \rightarrow X_{\infty}$ is any resolution of singularities, with $X_{s}^{\prime}=\sum_{i \in I} N_{i} E_{i}$, then this limit is given explicitly by

$$
\mathbb{L}^{-m} \sum_{\emptyset \neq J \subset I}(1-\mathbb{L})^{|J|-1}\left[\widetilde{E}_{J}^{o}\right]
$$

in $\mathcal{M}_{X_{s}}$.

Proof. This follows immediately from the computation in Corollary 7.7, and the remark preceding Corollary 7.8 .

Definition 8.3. The motivic volume

$$
S\left(X_{\infty} ; \widehat{K^{s}}\right) \in \mathcal{M}_{X_{s}}
$$

is by definition the limit of $-S\left(X_{\infty}, \omega ; T\right)$ for $T \rightarrow \infty$, where $\omega$ is any gauge form on $X_{\eta}$.

When $V$ is a locally closed subset of $X_{s}$, we define the motivic volume $S_{V}\left(X_{\infty} ; \widehat{K^{s}}\right)$ with support in $V$ as the image of $S\left(X_{\infty} ; \widehat{K^{s}}\right)$ under the base change morphism

$$
\mathcal{M}_{X_{s}} \rightarrow \mathcal{M}_{V}
$$

Finally, we define the motivic volume

$$
S\left(X_{\eta} ; \widehat{K^{s}}\right) \in \mathcal{M}_{k}
$$

as the image of $S\left(X_{\infty} ; \widehat{K^{s}}\right)$ under the forgetful morphism $\mathcal{M}_{X_{s}} \rightarrow \mathcal{M}_{k}$. It only depends on $X_{\eta}$, and not on $X_{\infty}$.

Remark. For these definitions, it is not necessary that $X_{\eta}$ admits a global gauge form. By additivity of the motivic integral w.r.t. open covers of $X_{\infty}$, we can use a system of local gauge forms on $X_{\eta}$, whose domains cover $X_{\eta}$. Since the limit of $-S\left(X_{\infty}, \omega ; T\right)$ for $T \rightarrow \infty$ does not depend on $\omega$, these local gauge forms do not have to coincide on the intersections of their domains. See also [39], Remark 6.5 .

Ayoub constructs in [5] a triangulated category of motives for rigid varieties $X_{\eta}$ over $k((t))$, and he shows that this category is equivalent to a certain subcategory of the stable homotopy-category of schemes over the torus $\mathbb{G}_{m, k}$. Pull-back via the unit section of $\mathbb{G}_{m, k}$ yields a motive over $k$, and this construction can be used to describe Ayoub's motivic nearby cycle functor. It seems very plausible that Ayoub's motive for $X_{\eta}$ coincides with our motivic volume $S\left(X_{\eta} ; \widehat{K^{s}}\right)$ in an appropriate Grothendieck ring of motives. Moreover, any reasonably defined additive invariant of $\bar{X}_{\eta}$ (e.g. its Hodge polynomial) should be computable on $S\left(X_{\eta} ; \widehat{K^{s}}\right)$.

A priori, $S\left(X_{\infty} ; \widehat{K^{s}}\right)$ depends not only on $X_{\infty} \widehat{\times}_{R} \widehat{R^{s}}$, but also on $X_{\infty}$. The following Proposition shows that, for any proper subscheme $Z$ of $X_{s}, \chi_{t o p}\left(S_{Z}\left(X_{\infty} ; \widehat{K^{s}}\right)\right)$ depends only on the $\widehat{K^{s}}$-analytic space $\bar{Z}$, and not on the embedding of this space in $X_{\infty} \widehat{\times}_{R} \widehat{R^{s}}$, if we assume that $X_{\infty}$ is algebrizable.

Proposition 8.4. Suppose $k$ is algebraically closed. Let $X$ be a generically smooth flat $R$-variety. Let $Z$ be a proper subvariety of the special fiber $X_{s}$. Then

$$
\chi_{\text {top }}\left(S_{Z}\left(\widehat{X} ; \widehat{K^{s}}\right)\right)=\chi_{e ́ t}(\overline{] Z[}),
$$


where $\chi_{\text {ét }}$ is the Euler characteristic associated to Berkovich' étale $\ell$-adic cohomology for non-archimedean analytic spaces.

Proof. Let $\varphi$ be a topological generator of the absolute Galois group $G\left(K^{s} / K\right)$. By definition,

$$
\chi_{\text {top }}\left(S_{Z}\left(\widehat{X} ; \widehat{K^{s}}\right)\right)=-\lim _{T \rightarrow \infty} \chi_{\text {top }}\left(S_{Z}(\widehat{X} ; T)\right)
$$

Hence, by our Trace Formula in Theorem 5.4 .

$$
\chi_{\text {top }}\left(S_{Z}\left(\widehat{X} ; \widehat{K^{s}}\right)\right)=-\lim _{T \rightarrow \infty} \sum_{n>0} \operatorname{Tr}\left(\varphi^{n} \mid H(\overline{] Z[})\right) T^{n}
$$

Recall the identity [18, 1.5.3]

$$
\begin{aligned}
\sum_{n>0} \operatorname{Tr}\left(F^{n}, V\right) T^{n} & =T \frac{d}{d T} \log \left(\operatorname{det}(1-T F, V)^{-1}\right) \\
& =-\frac{T \frac{d}{d T}(\operatorname{det}(1-T F, V))}{\operatorname{det}(1-T F, V)}
\end{aligned}
$$

for any endomorphism $F$ on a finite dimensional vector space $V$. Taking limits yields

$$
-\lim _{T \rightarrow \infty} \sum_{n>0} \operatorname{Tr}\left(F^{n}, V\right) T^{n}=\operatorname{dim}(V) .
$$

Applying this to $F=\varphi$ and $V=H(\overline{] Z[})$ yields

$$
\chi_{\text {top }}\left(S_{Z}\left(\widehat{X}, \widehat{K^{s}}\right)\right)=\chi_{e ́ t}(\overline{] Z}) .
$$

\section{Applications to motivic zeta functions and the Milnor fibration}

9.1. The analytic Milnor fiber. Let $X_{\infty}$ be a flat stft formal $R$-scheme. For any closed point $x$ on the special fiber $X_{s}$, the tube ] $x$ [ of $x$ in $X_{\infty}$ is an open rigid subspace of $X_{\eta}$. We will call it the analytic Milnor fiber of $X_{\infty}$ at $x$, and denote it by $\mathscr{F}_{x}$. As a $K$-rigid space, it is canonically isomorphic to the generic fiber of the completion $\widehat{X_{\infty} / x}$ of $X_{\infty}$ along $x$ (see [8, 0.2.7]). If $R=k[[t]]$, and $X_{\infty}$ is the $t$-adic completion a morphism of $k$-varieties $f: Z \rightarrow \operatorname{Spec} k[t]$, we will also call $\mathscr{F} x$ the analytic Milnor fiber of $f$ at $x$.

9.1.1. Cohomology of the analytic Milnor fiber. The following proposition shows that $\mathscr{F}_{x}$ has the "right" étale cohomology.

Lemma 9.1. Suppose that $k$ is algebraically closed. Let $X$ be a flat $R$-variety, and let $\mathcal{F}_{x}$ be the analytic Milnor fiber of $\widehat{X}$ at a closed point $x$ of $X_{s}$. Let $F$ be a constructible étale torsion sheaf on the generic fiber $X \times_{R} K$ of the $R$-scheme $X$, with torsion orders prime to $p$. Let $\hat{F}$ be the induced sheaf on the analytic space $X_{\eta} \widehat{\times}_{K} \widehat{K^{t}}$. The étale cohomology space $H^{q}\left(\mathscr{F}_{x} \widehat{\times} \widehat{K^{t}}, \hat{F}\right)$ is canonically isomorphic to the q-th cohomology space $R^{q} \psi_{\eta}^{t}(F)_{x}$ of the stalk at $x$ of the complex of tame nearby cycles $R \psi_{\eta}^{t}(F)$ of $X / R$, and this isomorphism is compatible with the geometric monodromy action of $G\left(K^{t} / K\right)$.

Proof. This follows from the comparison result in [7, 3.5]. 
Theorem 9.2. Let $X$ be a smooth, irreducible $\mathbb{C}$-variety, and consider a dominant morphism $f: X \rightarrow \mathbb{A}_{\mathbb{C}}^{1}=\operatorname{Spec} \mathbb{C}[t]$. Let $x$ be a point of the hypersurface $X_{s}$ defined by $f$, denote by $F_{x}$ the canonical topological Milnor fiber of $f$ at $x$, and by $M$ the monodromy automorphism on the singular cohomology $H_{\text {sing }}\left(F_{x}, \mathbb{C}\right) 1$.

Let $\mathscr{F}_{x}$ be the analytic Milnor fiber of $f$ at $x$, and let $\varphi$ be the canonical topological generator of $G\left(\mathbb{C}((t))^{s} / \mathbb{C}((t))\right) \cong \hat{\mathbb{Z}}(1)(\mathbb{C})$. Fix an embedding of $\mathbb{Q}_{\ell}$ in $\mathbb{C}$. There are canonical isomorphisms

$$
H_{\text {sing }}^{i}\left(F_{x}, \mathbb{C}\right) \cong H^{i}\left(\mathscr{F}_{x} \widehat{\times} \widehat{\mathbb{C}((t))^{s}}, \mathbb{Q}_{\ell}\right) \otimes_{\mathbb{Q}_{\ell}} \mathbb{C}
$$

compatible with the action of $M$ and $\varphi$.

Proof. By Lemma 9.1, we have, for each integer $n>0$, canonical $G\left(\mathbb{C}((t))^{s} / \mathbb{C}((t))\right)$ equivariant isomorphisms

$$
H^{i}\left(\mathscr{F}_{x} \widehat{\times} \widehat{\mathbb{C}((t))^{s}}, \mathbb{Z} / \ell^{n}\right) \cong R^{i} \psi_{\eta}^{t}\left(\mathbb{Z} / \ell^{n}\right)_{x}
$$

By [2, XIV], there exist canonical isomorphisms

$$
R^{i} \psi_{\eta}^{t}\left(\mathbb{Z} / \ell^{n}\right)_{x} \cong R^{i} \psi_{\eta}^{a n}\left(\mathbb{Z} / \ell^{n}\right)_{x}
$$

where $R \psi_{\eta}^{a n}$ is the complex of analytic vanishing cycles, and these isomorphisms are compatible with the action of $G\left(\mathbb{C}((t))^{s} / \mathbb{C}((t))\right)=\hat{\mathbb{Z}}(1)(\mathbb{C})$ and $\pi_{1}\left(\mathbb{G}_{m, \mathbb{C}}\right)=\mathbb{Z}$. Finally, there are canonical isomorphisms

$$
R^{i} \psi_{\eta}^{a n}\left(\mathbb{Z} / \ell^{n}\right)_{x} \cong H_{\text {sing }}^{i}\left(F_{x}, \mathbb{Z} / \ell^{n}\right)
$$

respecting the action of $\pi_{1}\left(\mathbb{G}_{m, \mathbb{C}}\right)$ (see e.g. [33],(8.11.7)). Taking projective limits over $n$ yields

$$
\begin{aligned}
H^{i}\left(\mathscr{F}_{x} \widehat{\times} \widehat{\mathbb{C}((t))^{s}}, \mathbb{Z}_{\ell}\right) & :=\lim _{\longleftarrow} H^{i}\left(\mathscr{F} x \widehat{\times} \widehat{\mathbb{C}((t))^{s}}, \mathbb{Z} / \ell^{n}\right) \\
& \cong \varliminf_{\lim } H_{\text {sing }}^{i}\left(F_{x}, \mathbb{Z} / \ell^{n}\right) \\
& =H_{\text {sing }}^{i}\left(F_{x}, \mathbb{Z}_{\ell}\right)
\end{aligned}
$$

Tensoring with $\mathbb{C}$ yields the required result.

As a corollary, we recover some classical results concernig the cohomology of the Milnor fiber. Let $X$ be a smooth irreducible variety over $\mathbb{C}$, and let $f: X \rightarrow \mathbb{A}_{\mathbb{C}}^{1}=$ Spec $\mathbb{C}[t]$ be a dominant morphism. Let $x$ be a complex point of the hypersurface $X_{s}$ defined by $f$. Let $h: X^{\prime} \rightarrow X$ be an embedded resolution of singularities for $f$, with $(f \circ h)=\sum_{i \in I} N_{i} E_{i}$. Let $F_{x}$ be the topological Milnor fiber at $x$, let $M$ be the monodromy transformation on the singular cohomology $H_{\text {sing }}\left(F_{x}, \mathbb{C}\right)$, and denote by $\zeta_{x}(T)$ the monodromy zeta function at $x$ (i.e. the alternating product of the characteristic polynomials of $M$ ).

Corollary $9.3\left([3)\right.$. If $x$ is a singular point of $f$, then $\operatorname{Tr}\left(M \mid H_{\text {sing }}\left(F_{x}, \mathbb{C}\right)\right)=0$. Else, $\operatorname{Tr}\left(M \mid H_{\text {sing }}\left(F_{x}, \mathbb{C}\right)\right)=1$.

Proof. If $Y$ is any regular, flat $R$-variety, then $S m(\widehat{Y}) \rightarrow \widehat{Y}$ is a Néron $R$-smoothening, by [11, $\S 3$, Prop 2]. Hence, $S_{x}(\widehat{X})=0$ if $x$ is a singular point of $f$, and $S_{x}(\widehat{X})=1$ else. Now our trace formula in Theorem 5.4 yields the result.

\footnotetext{
${ }^{1}$ We recalled the definitions in the introduction.
} 
Corollary 9.4 (A'Campo's formula [4]).

$$
\begin{aligned}
\operatorname{Tr}\left(M^{d} \mid H_{\text {sing }}\left(F_{x}, \mathbb{C}\right)\right) & =\sum_{N_{i} \mid d} N_{i} \chi_{\text {top }}\left(E_{i}^{o} \cap h^{-1}(x)\right), \\
\zeta_{x}(T) & =\prod_{i}\left(T^{N_{i}}-1\right)^{-\chi_{t o p}\left(E_{i}^{o} \cap h^{-1}(x)\right)} .
\end{aligned}
$$

Proof. The second formula follows from the first via the identity (11) in Section 8 .

By Theorem [5.4, the expressions are valid if we replace $F_{x}$ by $\mathscr{F}_{x} \widehat{\times} \widehat{\mathbb{C}((t))^{s}}$, where $\mathscr{F}_{x}$ is the analytic Milnor fiber $\mathscr{F}_{x}$ at $x$, and if we replace singular cohomology by Berkovich' étale cohomology for analytic spaces.

The expression now follows from the comparison result in Theorem 9.2 ,

9.1.2. Points of the analytic Milnor fiber. We suppose that $R=k[[t]]$, with $k$ an algebraically closed field of characteristic zero. Let $X$ be any variety over $k$, and consider a $k$-morphism $f: X \rightarrow \operatorname{Spec} k[t]$, flat over the origin. Denote by $\widehat{X}$ the $t$-adic completion of $f$. We can describe the points of the generic fiber $X_{\eta}$, and the specialization map $s p:\left|X_{\eta}\right| \rightarrow|\widehat{X}|=\left|X_{s}\right|$ on the level of the underlying sets.

We denote by $\mathcal{L}(X)$ the arc scheme of $X$, as defined in [22, p.1]. It is a scheme over $k$, of infinite type in general, and for any field $k^{\prime}$ containing $k$,

$$
\mathcal{L}(X)\left(k^{\prime}\right)=\operatorname{Hom}_{k}\left(\operatorname{Spec} k^{\prime}[[u]], X\right)
$$

Reduction modulo $u$ yields a projection morphism of $k$-schemes $\pi_{0}: \mathcal{L}(X) \rightarrow X$.

For any integer $d>0$, we denote by $\mathcal{X}(d)$ the closed subscheme of $\mathcal{L}(X)$ defined by

$$
\mathcal{X}(d)=\left\{\psi \in \mathcal{L}(X) \mid f(\psi)=u^{d}\right\}
$$

We will construct a canonical bijection

$$
\varphi: X_{\eta}(K(d)) \rightarrow \mathcal{X}(d)(k)
$$

such that the square

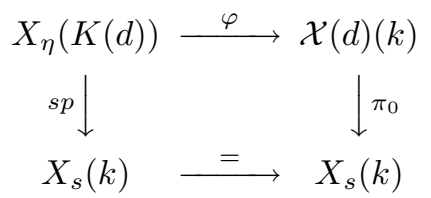

commutes.

The specialization morphism of ringed sites $s p: X_{\eta} \rightarrow \widehat{X}$ induces a bijection $X_{\eta}(K(d)) \rightarrow \widehat{X}(R(d))$, and the morphism $s p: X_{\eta}(K(d)) \rightarrow X_{s}(k)$ maps a point of $X_{\eta}(K(d))$ to the reduction modulo $t_{d}$ of the corresponding point of $\widehat{X}(R(d))$.

By Grothendieck's Existence Theorem [26, 5.4.1], the completion functor induces a bijection $\left(X \times_{k[t]} R\right)(R(d)) \rightarrow \widehat{X}(R(d))$. A reparametrization $t \mapsto t_{d}$ yields a bijection $\left(X \times_{k[t]} R\right)(R(d)) \rightarrow \mathcal{X}(d)(k)$.

Hence, for any closed point $x$ of $X_{s}$, the set $\mathscr{F}_{x}(K(d))$ corresponds bijectively to the fiber of $\pi_{0}: \mathcal{X}(d)(k) \rightarrow X_{s}(k)$ over $x$. In other words, a $K(d)$-point of $\mathscr{F}_{x}$ is nothing but an arc $\psi \in H^{\prime} m_{k}(\operatorname{Spec} k[[u]], X)$ with $f(\psi(u))=u^{d}$ and $\psi(0)=x$.

The Galois group $G(K(d) / K)=\mu_{d}(k)$ acts on $X_{\eta}(K(d))$ as follows: if $\psi$ is an element of $\mathcal{X}(d)(k)$, an element $\xi \in \mu_{d}(k)$ acts on $\psi$ by $\xi * \psi(u)=\psi(\xi \cdot u)$. 
9.2. The local singular series and the Gelfand-Leray form. Let $k$ be a field of characteristic zero, let $X$ be a smooth irreducible variety over $k$, of dimension $m$, and let $f: X \rightarrow \mathbb{A}_{k}^{1}=\operatorname{Spec} k[t]$ be a dominant morphism, smooth on the complement of the special fiber $X_{s}$ of $f$ in $X$. Denote by $\widehat{X}$ the $t$-adic completion of $X$, and by $X_{\eta}$ its generic fiber. Let $\omega$ be a gauge form on $X$. The Koszul complex associated to $d f$ is exact on $V:=X \backslash X_{s}$, and in particular

$$
\Omega_{V}^{m-2} \stackrel{\wedge d f}{\longrightarrow} \Omega_{V}^{m-1} \stackrel{\wedge d f}{\longrightarrow} \Omega_{V}^{m} \longrightarrow 0
$$

is an exact sequence of sheaves on $V$. This means that we can choose, for each point $y$ in $V$, an element $\alpha_{y}$ in $\left(\Omega_{V}^{m-1}\right)_{y}$, such that $(d f)_{y} \wedge \alpha_{y}=\omega_{y}$. Since the $\alpha_{y}$ are unique up to a term $d f \wedge *$, they glue together to a well-defined global section

$$
\frac{\omega}{d f} \in \Omega_{V / \mathbb{A}_{k}^{1}}^{m-1}(V)
$$

This relative differential form induces a gauge form $\frac{\omega}{d f}$ on $X_{\eta}$.

Definition 9.5. We call

$$
\frac{\omega}{d f} \in \Omega_{X_{\eta} / K}^{m-1}\left(X_{\eta}\right)
$$

the Gelfand-Leray form of $\omega$ w.r.t. $f$.

Lemma 9.6. Let $h: X^{\prime} \rightarrow X$ be an embedded resolution of singularities for the morphism $f$, with $X_{s}^{\prime}=\sum_{i \in I} N_{i} E_{i}$ and relative canonical divisor $K_{X^{\prime} / X}=$ $\sum_{i \in I}\left(\nu_{i}-1\right) E_{i}$. Then

$$
\operatorname{ord}_{E_{i}} h^{*}\left(\frac{\omega}{d f}\right)=\nu_{i}-N_{i}
$$

for any $i \in I$.

Proof. The pullback $h^{*}(\omega / d f)$ coincides with the Gelfand-Leray form associated to $h^{*}(\omega)$ and $f \circ h$, since for any differential form $\alpha$ on $X$, we have

$$
h^{*} \alpha \wedge d(f \circ h)=h^{*} \alpha \wedge h^{*} d f=h^{*}(\alpha \wedge d f) .
$$

Choosing local coordinates $\left(x_{1}, \ldots, x_{m}\right)$ and $\left(x_{1}^{\prime}, \ldots, x_{m}^{\prime}\right)$ on $X$, resp. $X^{\prime}$, we may assume that $f \circ h=u\left(x_{1}^{\prime}\right)^{N_{i}}$, with $u$ a unit, and that the Jacobian of $h$ is given by $J a c_{h}=u^{\prime}\left(x_{1}\right)^{\nu_{i}-1}$, for some unit $u^{\prime}$. This means that the pullback $h^{*} \omega$ is given by $\left(x_{1}\right)^{\nu_{i}-1}$ times a gauge form on $X^{\prime}$. Passing to an étale cover of $X^{\prime}$, we may assume that $u=1$, by Lemma 6.9. In this case, $h^{*} \omega / d(f \circ h)$ equals $\left(x_{1}^{\prime}\right)^{\nu_{i}-N_{i}}$ times a nowhere vanishing relative form on $\widehat{X^{\prime}} / R$, hence $\operatorname{ord}_{E_{i}}\left(h^{*} \omega / d(f \circ h)\right)=\nu_{i}-N_{i}$.

Corollary 9.7. We have, for any $d>0$,

$$
F\left(\widehat{X}, \frac{\omega}{d f} ; d\right)=\mathbb{L}^{-(m-1)} \sum_{\emptyset \neq J \subset I}(\mathbb{L}-1)^{|J|-1}\left[\widetilde{E}_{J}^{o}\right]\left(\sum_{\substack{k_{i} \geq 1, i \in J \\ \sum_{i \in J} k_{i} N_{i}=d}} \mathbb{L}^{k_{i}\left(N_{i}-\nu_{i}\right)}\right) \in \mathcal{M}_{X_{s}}
$$

In particular, the right hand side does not depend on the choice of $\omega$.

If $d$ is not $X_{s}^{\prime}$-linear, then the above expression reduces to

$$
F\left(\widehat{X}, \frac{\omega}{d f} ; d\right)=\mathbb{L}^{-(m-1)} \sum_{N_{i} \mid d}\left[\widetilde{E}_{i}^{o}\right] \mathbb{L}^{d-d \nu_{i} / N_{i}} \in \mathcal{M}_{X_{s}}
$$

Proof. This follows immediately from Theorem 7.6 . 
MOTIVIC SERRE INVARIANTS, RAMIFICATION, AND THE ANALYTIC MILNOR FIBER31

Definition 9.8. We define the local singular series associated to $f$ by

$$
F(f ; d):=F\left(\widehat{X}, \frac{\omega}{d f} ; d\right)
$$

where $\omega$ is any gauge form on $X$.

Remark. For this definition, it is not necessary that $X$ admits a global gauge form $\omega$. See Remark 8 .

9.3. Comparison to the motivic zeta function. Let $k$ be a field of characteristic zero, let $X$ be a smooth irreducible $k$-variety of dimension $m$, and let $f: X \rightarrow \mathbb{A}_{k}^{1}=$ Spec $k[t]$ be a dominant morphism.

As in [22, p.1], we denote, for any integer $d>0$, by $\mathcal{L}_{d}(X)$ the $k$-scheme representing the functor

$$
(k-\text { algebras }) \rightarrow(\text { Sets }): A \mapsto H_{k}\left(\operatorname{Spec} A[u] /\left(u^{d+1}\right), X\right)
$$

Following [23, 3.2], we denote by $\mathcal{X}_{d, 1}$ the $X_{s}$-variety

$$
\mathcal{X}_{d, 1}=\left\{\psi \in \mathcal{L}_{d}(X) \mid f(\psi(u))=u^{d} \bmod u^{d+1}\right\}
$$

where the structural morphism $\mathcal{X}_{d, 1} \rightarrow X_{s}$ is given by reduction modulo $u$. In 23 , 3.2.1], the motivic zeta function $Z(T)$ of $f$ is defined as

$$
Z(T)=\sum_{d=1}^{\infty}\left[\mathcal{X}_{d, 1}\right] \mathbb{L}^{-m d} T^{d} \in \mathcal{M}_{X_{s}}[[T]]
$$

Actually, the coefficients live in a more refined Grothendieck ring, as we will see in Section 9.4

In loc. cit., Denef and Loeser show that $Z(T)$ is rational over $\mathcal{M}_{X_{s}}$, and they give an explicit expression in terms of an embedded resolution of $f$ (see also Section 9.4).

Lemma 9.9. Let $X$ be a smooth irreducible variety over $k$ of dimension $m$, let $f: X \rightarrow \mathbb{A}_{k}^{1}$ be a dominant morphism, and let $\omega$ be a gauge form on $X$. We have

$$
F\left(\widehat{X}, \frac{\omega}{d f} ; d\right)=\mathbb{L}^{-(d+1)(m-1)}\left[\mathcal{X}_{d, 1}\right]
$$

in $\mathcal{M}_{X_{s}}$, for each integer $d>0$.

Proof. Take an embedded resolution $X^{\prime} \rightarrow X$ for $f$, such that $d$ is not $X_{s}^{\prime}$-linear, and combine Corollary 9.7 with the computation in [24, Theorem 2.4.

Theorem 9.10. Let $X$ be a smooth irreducible variety over $k$ of dimension $m$, let $f: X \rightarrow \mathbb{A}_{k}^{1}$ be a dominant morphism, and let $\omega$ be any gauge form on $X$. We have

$$
S\left(\widehat{X}, \frac{\omega}{d f} ; T\right)=\mathbb{L}^{-(m-1)} Z(\mathbb{L} T) \in \mathcal{M}_{X_{s}}[[T]]
$$

Proof. This follows immediately from Lemma 9.9.

Hence, we recover the motivic zeta function as a Mellin transform of the local singular series associated to $f$. Our trace formula yields the following cohomological interpretation of the motivic zeta function. 
Theorem 9.11. Suppose that $k$ is algebraically closed. Let $Z$ be a subvariety of $X_{s}$, proper over $k$, and denote by $\mathcal{X}_{d, 1, Z}$ the fiber of $\mathcal{X}_{d, 1} \rightarrow X_{s}$ over $Z$. Let $\varphi$ be a topological generator of the geometric monodromy group $G\left(K^{s} / K\right)$. For any integer $d>0$,

$$
\chi_{\text {top }}\left(\mathcal{X}_{d, 1, Z}\right)=\operatorname{Tr}\left(\varphi^{d} \mid H(\overline{] Z[})\right) .
$$

Proof. This is an immediate consequence of Lemma 9.9, and the trace formula Theorem 5.4 .

We recover the main result of Denef and Loeser's paper [24].

Corollary 9.12 ([24], Theorem 1.1). If $k=\mathbb{C}$, we get, for each complex point $x$ on $X_{s}$, and each $d>0$,

$$
\chi_{\text {top }}\left(\mathcal{X}_{d, 1, x}\right)=\operatorname{Tr}\left(M^{d} \mid H_{\text {sing }}\left(F_{x}, \mathbb{C}\right)\right),
$$

where $F_{x}$ is the canonical topological Milnor fiber of $f$ at $x$, and $M$ is the monodromy transformation.

Proof. This follows from the comparison result in Theorem 9.2 and from Theorem 9.11 above.

9.4. The motivic Milnor fiber. We recall Denef and Loeser's definition of the motivic Milnor fiber [23, 3.5]. Let $k$ be an algebraically closed field of characteristic zero, let $X$ be a smooth irreducible $k$-variety of dimension $m$, and let $f: X \rightarrow$ $\mathbb{A}_{k}^{1}=\operatorname{Spec} k[t]$ be a dominant morphism. We denote the hypersurface defined by $f$ in $X$ by $X_{s}$.

Let $\mu$ be the inverse limit of the groups $\mu_{d}(k)$ of $d$-th roots of unity in $k$. Let $S$ be a variety over $k$. By a good $\mu$-action on an algebraic variety $Z$ over $S$, we mean an action of $\mu$ on $Z$, equivariant w.r.t. the structure morphism $Z \rightarrow S$ (where $S$ carries the trivial action), that factors through some $\mu_{d}$, and such that each orbit is contained in an affine open subscheme of $Z$. The relative equivariant Grothendieck ring $K_{0}^{\mu}\left(\operatorname{Var}_{S}\right)$ of $S$-varieties with good $\mu$-action is defined in [23, 2.4].

For each $d>0$, the $X_{s}$-variety $\mathcal{X}_{d, 1}$ carries a good $\mu$-action, defined as follows: if $\xi$ is an element of $\mu_{d}(k)$, and $\psi(u)$ is an element of $\mathcal{X}_{d, 1}$, then $\xi * \psi=\psi(\xi \cdot u)$. Denef and Loeser define the equivariant motivic zeta function as

$$
Z^{m o n}(T)=\sum_{d=1}^{\infty}\left[\mathcal{X}_{d, 1}\right] \mathbb{L}^{-m d} T^{d} \in K_{0}^{\mu}\left(\operatorname{Var}_{X_{s}}\right)
$$

They show it is rational over $\mathcal{M}_{X_{s}}^{\mu}=K_{0}^{\mu}\left(\operatorname{Var}_{X_{s}}\right)\left[\mathbb{L}_{X_{s}}^{-1}\right]$, where $\mathbb{L}_{X_{s}}$ denotes the class of the affine line over $X_{s}$, with trivial $\mu$-action.

In fact, they obtain an explicit formula in terms of an embedded resolution of singularities for $f$ on $X$. Let $h: X^{\prime} \rightarrow X$ be an embedded resolution for $f$, with $X_{s}^{\prime}=\sum_{i \in I} N_{i} E_{i}$, and with relative canonical divisor $K_{X^{\prime} \mid X}=\sum_{i \in I}\left(\nu_{i}-1\right) E_{i}$. For any non-empty subset $J$ of $I$, the cover $\widetilde{E}_{J}^{o} / E_{J}^{o}$ (defined in Section 4 ) is Galois with group $\mu_{m_{J}}(k)$.

By [23, Theorem 3.3.1, we have

$$
Z^{m o n}(T)=\sum_{\emptyset \neq J \subset I}(\mathbb{L}-1)^{|J|-1}\left[\widetilde{E}_{J}^{o}\right] \prod_{i \in J} \frac{\mathbb{L}^{-\nu_{i}} T^{N_{i}}}{1-\mathbb{L}^{-\nu_{i}} T^{N_{i}}} \text { in } \mathcal{M}_{X_{s}}^{\mu}[[T]] .
$$


Inspired by the $p$-adic case 20, Denef and Loeser defined the motivic nearby cycles $\mathcal{S}_{f}$ by taking formally the limit of $-Z^{m o n}(T)$ for $T \rightarrow \infty$, i.e.

$$
\mathcal{S}_{f}=\sum_{\emptyset \neq J \subset I}(1-\mathbb{L})^{|J|-1}\left[\widetilde{E}_{J}^{o}\right] \in \mathcal{M}_{X_{s}}^{\mu} .
$$

This terminology is justified by the fact that, when $k=\mathbb{C}$, for each closed point $x$ of $X_{s}$, the mixed Hodge structure of the fiber $\mathcal{S}_{f, x} \in \mathcal{M}_{k}^{\mu}$ of $\mathcal{S}_{f}$ over $x$ coincides with the mixed Hodge structure of the Milnor fiber of $f$ at $x$ (in an appropriate Grothendieck group of mixed Hodge structures), and the $\mu$-action corresponds to the semi-simple part of the monodromy action [21, 4.2]. Denef and Loeser called $\mathcal{S}_{f, x}$ the motivic Milnor fiber of $f$ at $x$.

Theorem 9.13. Denoting the $t$-adic completion of $X$ by $\widehat{X}$, we have

$$
S\left(\widehat{X} ; \widehat{K^{s}}\right)=\mathbb{L}^{-(m-1)} \mathcal{S}_{f} \in \mathcal{M}_{X_{s}}
$$

For any closed point $x$ on $X_{s}$, we have

$$
S_{x}\left(\widehat{X} ; \widehat{K^{s}}\right)=\mathbb{L}^{-(m-1)} \mathcal{S}_{f, x} \in \mathcal{M}_{x}
$$

Proof. This follows from Theorem 9.10

Hence, we recover Denef and Loeser's motivic nearby cycles as the motivic volume of the rigid "nearby fiber" $X_{\eta}$, and in some sense, we recover the motivic Milnor fiber $\mathcal{S}_{f, x}$ as the motivic volume of the analytic Milnor fiber $\mathcal{F}_{x}$.

\section{ACKNOWLedGements}

The authors would like to thank François Loeser for inspiring discussions. The first author is indebted to L. Illusie, V. Berkovich, and O. Villamayor, who were so kind to answer his questions on nearby cycles, non-archimedean spaces, and resolution of singularities.

\section{REFERENCES}

[1] Groupes de monodromie en géométrie algébrique. I. Springer-Verlag, Berlin, 1972. Séminaire de Géométrie Algébrique du Bois-Marie 1967-1969 (SGA 7 I), Dirigé par A. Grothendieck. Avec la collaboration de M. Raynaud et D. S. Rim, Lecture Notes in Mathematics, Vol. 288.

[2] Groupes de monodromie en géométrie algébrique. II. Springer-Verlag, Berlin, 1973. Séminaire de Géométrie Algébrique du Bois-Marie 1967-1969 (SGA 7 II), Dirigé par P. Deligne et N. Katz, Lecture Notes in Mathematics, Vol. 340.

[3] N. A'Campo. Le nombre de Lefschetz d'une monodromie. Indag. Math., 35:113-118, 1973.

[4] N. A'Campo. La fonction zêta d'une monodromie. Comment. Math. Helvetici, 50:233-248, 1975.

[5] J. Ayoub. Motives of rigid varieties. Lecture at the Conference on Hodge Theory, Venice, 19-24 June 2006.

[6] V. G. Berkovich. Étale cohomology for non-Archimedean analytic spaces. Publ. Math., Inst. Hautes Étud. Sci., 78:5-171, 1993.

[7] V. G. Berkovich. Vanishing cycles for formal schemes II. Invent. Math., 125(2):367-390, 1996.

[8] P. Berthelot. Cohomologie rigide et cohomologie rigide à supports propres. Prepublication, Inst. Math. de Rennes, 1996.

[9] S. Bosch. Lectures on formal and rigid geometry. preprint, http://www.math1.unimuenster.de/sfb/about/publ/bosch.html, 2005.

[10] S. Bosch, U. Güntzer, and R. Remmert. Non-Archimedean analysis. A systematic approach to rigid analytic geometry., volume 261 of Grundlehren der Mathematischen Wissenschaften. Springer Verlag, 1984. 
[11] S. Bosch, W. Lütkebohmert, and M. Raynaud. Néron models, volume 21. Ergebnisse der Mathematik und ihrer Grenzgebiete, 1990.

[12] S. Bosch, W. Lütkebohmert, and M. Raynaud. Formal and rigid geometry. III: The relative maximum principle. Math. Ann., 302(1):1-29, 1995.

[13] S. Bosch and K. Schlöter. Néron models in the setting of formal and rigid geometry. Math. Ann., 301(2):339-362, 1995.

[14] A. Bravo, S. Encinas, and O. Villamayor. A simplified proof of desingularization and applications. Rev. Mat. Iberoamericana, 21(2):349-458, 2005.

[15] R. Cluckers and F. Loeser. Constructible motivic functions, integrals with parameters, and cell decomposition. arxiv:math.AG/0410203.

[16] B. Conrad. Irreducible components of rigid spaces. Ann. Inst. Fourier, 49(2):473-541, 1999.

[17] A. J. de Jong. Crystalline Dieudonné module theory via formal and rigid geometry. Publ. Math., Inst. Hautes Étud. Sci., 82:5-96, 1995.

[18] P. Deligne. La conjecture de Weil. I. Publ. Math., Inst. Hautes Étud. Sci., 43:273-307, 1973.

[19] J. Denef. Report on Igusa's local zeta function. In Séminaire Bourbaki, Vol. 1990/91, Exp. No.730-744, volume 201-203, pages 359-386, 1991.

[20] J. Denef. Degree of local zeta functions and monodromy. Compositio Math., 89:207-216, 1993.

[21] J. Denef and F. Loeser. Motivic Igusa zeta functions. J. Algebraic Geom., 7:505-537, 1998, arxiv:math.AG/9803040.

[22] J. Denef and F. Loeser. Germs of arcs on singular algebraic varieties and motivic integration. Invent. Math., 135:201-232, 1999, arxiv:math.AG/9803039.

[23] J. Denef and F. Loeser. Geometry on arc spaces of algebraic varieties. Progr. Math., 201:327348, 2001, arxiv:math.AG/0006050.

[24] J. Denef and F. Loeser. Lefschetz numbers of iterates of the monodromy and truncated arcs. Topology, 41(5):1031-1040, 2002.

[25] D. Eisenbud and J. Harris. The geometry of schemes, volume 197 of Graduate Texts in Mathematics. Springer-Verlag, New York, 2000.

[26] A. Grothendieck and J. Dieudonné. Eléments de Géométrie Algébrique, iii. Publ. Math., Inst. Hautes Étud. Sci., 11:5-167, 1961.

[27] A. Grothendieck and J. Dieudonné. Eléments de Géométrie Algébrique, iv, première partie. Publ. Math., Inst. Hautes Étud. Sci., 20:5-259, 1964.

[28] G. Guibert, F. Loeser, and M. Merle. Iterated vanishing cycles, convolution, and a motivic analogue of a conjecture of Steenbrink. Duke Math. J., 132(3):409-457, 2006.

[29] J. Igusa. An introduction to the theory of local zeta functions. Studies in Advanced Mathematics. AMS, 2000.

[30] L. Illusie. Théorie de Brauer et caractéristique d'Euler-Poincaré (d'après P. Deligne). In The Euler-Poincaré characteristic, volume 82 of Astérisque, pages 161-172. 1981.

[31] G. Kempf, F. Knudsen, D. Mumford, and B. Saint-Donat. Toroidal embeddings 1, volume 339 of Lecture Notes in Mathematics. Springer-Verlag, 1973.

[32] M. Kontsevich. Lecture at Orsay. December 7, 1995.

[33] V. Kulikov. Mixed Hodge Structures and Singularities, volume 132 of Cambridge Tracts in Mathematics. Cambridge University Press, 1998.

[34] F. Loeser and J. Sebag. Motivic integration on smooth rigid varieties and invariants of degenerations. Duke Math. J., 119:315-344, 2003.

[35] J. Milnor. Singular points of complex hypersurfaces, volume 61 of Annals of Math. Studies. Princeton University Press, 1968.

[36] J. Nicaise and J. Sebag. Rigid geometry and the monodromy conjecture. to appear in the Conference Proceedings of Singularités, CIRM, Luminy, 24 January-25 February 2005.

[37] J. Nicaise and J. Sebag. Invariant de Serre et fibre de Milnor analytique. C.R.Ac.Sci., 341(1):21-24, 2005.

[38] J. Nicaise and J. Sebag. Motivic Serre invariants and Weil restriction. preprint, 2006.

[39] J. Nicaise and J. Sebag. Motivic Serre invariants of curves. to appear in Manuscr. Math., 2006.

[40] B. Poonen. The Grothendieck ring of varieties is not a domain. Math. Res. Letters, 9(4):493498, 2002, arxiv:math.AG/0204306.

[41] B. Rodrigues and W. Veys. Poles of Zeta functions on normal surfaces. Proc. London Math. Soc., 87(3):164-196, 2003. 
MOTIVIC SERRE INVARIANTS, RAMIFICATION, AND THE ANALYTIC MILNOR FIBER35

[42] J. Sebag. Intégration motivique sur les schémas formels. Bull. Soc. Math. France, 132(1):1-54, 2004.

Université Lille 1, Laboratoire Painlevé, CNRS - UMR 8524, Cité Scientifique, 59 655 Villeneuve D'Ascq Cédex, France

E-mail address: johannes.nicaise@math.univ-lille1.fr

Université Bordeaux 1, IMB, Laboratoire A2X, 351 Cours de la libération, 33405 Talence cedex, France

E-mail address: julien.sebag@math.u-bordeaux1.fr 